\title{
Outage Probability Analysis of Cognitive Transmissions: Impact of Spectrum Sensing Overhead
}

\author{
Yulong Zou, Student Member, IEEE, Yu-Dong Yao, Senior Member, IEEE, and Baoyu Zheng, Member, IEEE
}

\begin{abstract}
In cognitive radio networks, a cognitive source node requires two essential phases to complete a cognitive transmission process: the phase of spectrum sensing with a certain time duration (also referred to as spectrum sensing overhead) to detect a spectrum hole and the phase of data transmission through the detected spectrum hole. In this paper, we focus on the outage probability analysis of cognitive transmissions by considering the two phases jointly to examine the impact of spectrum sensing overhead on system performance. A closed-form expression of an overall outage probability that accounts for both the probability of no spectrum hole detected and the probability of a channel outage is derived for cognitive transmissions over Rayleigh fading channels. We further conduct an asymptotic outage analysis in high signal-to-noise ratio regions and obtain an optimal spectrum sensing overhead solution to minimize the asymptotic outage probability. Besides, numerical results show that a minimized overall outage probability can be achieved through a tradeoff in determining the time durations for the spectrum hole detection and data transmission phases. In this paper, we also investigate the use of cognitive relay to improve the outage performance of cognitive transmissions. We show that a significant improvement is achieved by the proposed cognitive relay scheme in terms of the overall outage probability.
\end{abstract}

Index Terms-Cognitive radio, spectrum sensing, overhead, cognitive relay transmission, outage probability.

\section{INTRODUCTION}

$\mathbf{T}$ HERE are increasing demands for the wireless radio spectrum with the emergency of many new wireless communication networks (e.g., wireless local area networks, wireless sensor networks, Bluetooth and so on). Meanwhile, according to the Federal Communications Commission (FCC), large portions of the licensed wireless spectrum resources are

Manuscript received January 26, 2010; revised April 7, 2010; accepted May 28, 2010. The associate editor coordinating the review of this paper and approving it for publication was J. Olivier.

Y. Zou is with the Institute of Signal Processing and Transmission, Nanjing University of Posts and Telecommunications, Nanjing, Jiangsu 210003, China, and with the Electrical and Computer Engineering Department, Stevens Institute of Technology, Hoboken, NJ 07030, USA (e-mail: zouyulong198412@126.com, yzou1@stevens.edu).

Y.-D. Yao is with the Electrical and Computer Engineering Department, Stevens Institute of Technology, Hoboken, NJ 07030, USA (e-mail: yyao@stevens.edu).

B. Zheng is with the Institute of Signal Processing and Transmission, Nanjing University of Posts and Telecommunications, Nanjing, Jiangsu 210003, China (e-mail: zby@njupt.edu.cn).

This work was partially supported by the Postgraduate Innovation Program of Scientific Research of Jiangsu Province (Grant Nos. CX08B_080Z, CX09B_150Z) and the National Natural Science Foundation of China (Grant No. 60972039).

Digital Object Identifier 10.1109/TWC.2010.061710.100108 under utilized [1]. In order to address this issue, cognitive radio [2] has been proposed by allowing a cognitive user to access a spectrum hole (that is a frequency band licensed to a primary user but not utilized by that user at a particular time and a specific geographic location [3]), which promotes the efficient utilization of the licensed spectrum. As stated in [3], cognitive radio is an intelligent wireless communication system, which learns from its surrounding environment and adapts its internal states to statistical variations of the environment.

In cognitive radio networks, a cognitive source node typically requires two essential phases to complete its transmission to its destination: 1) a spectrum sensing phase (also known as a spectrum hole detection phase), in which the cognitive source attempts to detect an available spectrum hole with a certain time duration (referred to as spectrum sensing overhead), and 2) a data transmission phase, in which data is transmitted to the destination through the detected spectrum hole. The two phases have been studied individually in terms of different detection [5] - [13] or different transmission [14] - [22] techniques.

In spectrum sensing, the energy detection [5], [6] and the matched filter detection [7], [8] have been proposed first and investigated extensively. It has been shown that the energy detection can not differentiate signal types, which could lead to more false detections triggered by some unintended interference signals [5]. Although the matched filter is an optimal detector in stationary Gaussian noise scenario, it requires prior information of the primary user signal, such as the pulse shape, modulation type and so on [4]. As an alternative, the cyclostationary feature detector has been presented in [9], [10], which can differentiate the modulated signal from the interference and additive noise. The advantage of cyclostationary detection comes at the expense of high computational complexities since it requires an extra training process to extract significant features. Meanwhile, in order to combat fading effects, a collaborative spectrum sensing approach has been proposed [11], where the detection results from multiple cognitive users are pooled together at a fusion center by using a logic rule. Recently, in [12], [13], the authors have applied cooperative diversity [16], [17] to the detection of the primary user and shown that the detection time can be reduced greatly through the cooperation between the cognitive users.

In the wireless transmission research, a large number of studies are motivated to combat the large-scale and smallscale fading. Two wireless transmission technologies, i.e., the 
relay and the diversity techniques, have been proposed [14] - [23] in order to provide reliable systems with high datarates. As stated in [14], the relay technique has been generally considered as an effective method to improve the capacity and coverage for next-generation wireless networks. On the other hand, multiple-input and multiple-output (MIMO) [15] has been proposed as an effective diversity scheme that can increase the channel capacity greatly by employing multiple antennas at both the transmitter and receiver. In [16] - [23], the authors have put forward the cooperative diversity to implement virtual multiple antennas through the cooperation between the source and the relay nodes, which combines the advantages of the relay and diversity techniques. More recently, in [28], [29], the cooperative relay technology has also been explored at the medium access control (MAC) and higher layers to improve the system throughput of cognitive radio networks.

Notice that the spectrum hole detection and data transmission phases can not be designed and optimized in isolation since they could affect each other. For example, an available spectrum hole would get wasted if the cognitive source has not detected the hole within a certain time duration. This decreases the spectrum hole utilization efficiency. While increasing the time duration of the hole detection phase improves the detection probability of spectrum holes, it comes at the expense of a reduction in transmission performance since less time is now available for the data transmission. In [24], the authors have studied a tradeoff in optimizing the performance of the secondary user under a targeted level of the primary interference protection. In [25], [26], a sensing-throughput tradeoff has been investigated for cognitive radio networks, where the research focus is on the maximization of secondary throughput under the constraint of primary user protection. Besides, another optimal spectrum sensing framework has been explored in [27], where the optimization objective is to minimize the spectrum sensing time without considering the transmission link condition and, moreover, only an additive white Gaussian noise (AWGN) channel is considered for the sensing performance analysis. However, the transmission link condition plays an important role in optimizing the cognitive transmission performance. For example, if a small-scaled fading term is taken into account for the transmission link, an outage event may occur given a fixed transmit power and data transmission rate when the transmission link is in relatively deep fading. In this case, a longer time duration is needed for the data transmission phase to reduce the outage probability satisfying a predefined target.

The main contributions of this paper are described as follows. First, unlike the separate analysis of the spectrum hole detection and data transmission phases [5] - [23], we jointly consider the two parts to examine the impact of the spectrum sensing overhead on the overall system performance. Second, we focus on the minimization of outage probability of secondary transmissions under a required probability of detection of primary users over Rayleigh fading channels, differing from [24] - [27] where the research is to either minimize the spectrum sensing time or to maximize the secondary throughput. Third, we derive a closed-form expression of the overall outage probability over Rayleigh fading channels,

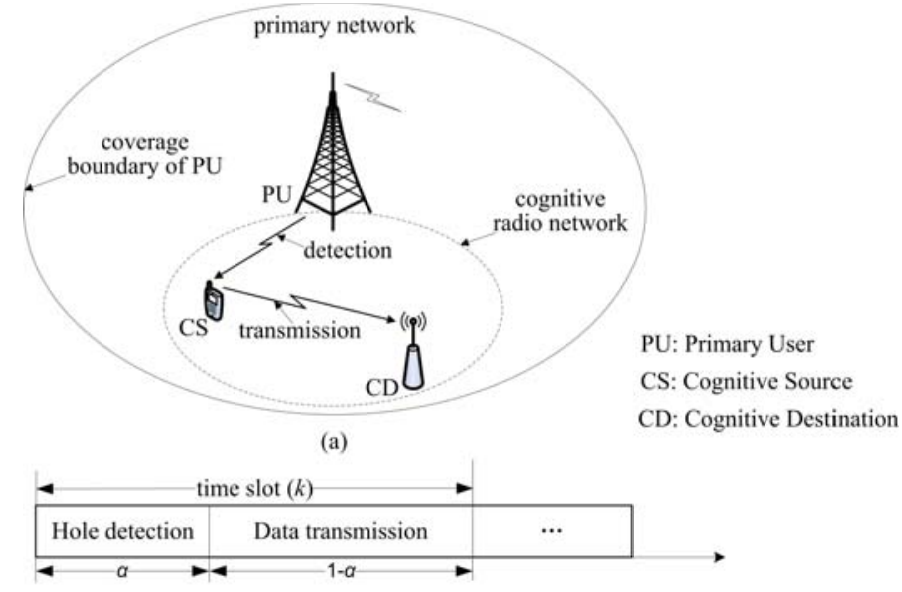

(b)

Fig. 1. (a) Coexistence of a primary wireless network and a cognitive radio network; (b) the allocation of time durations: hole detection versus data transmission.

which accounts for both the probability of no spectrum hole detected and the probability of a channel outage, for cognitive transmissions. Finally, in order to improve the system performance, we propose a cognitive relay transmission scheme and present its outage probability analysis over Rayleigh fading channels.

The remainder of this paper is organized as follows. Section II describes the system model of cognitive transmission that considers both the spectrum hole detection and data transmission phases, followed by the outage analysis in Section III, where the corresponding numerical evaluations are also provided to show the system performance of cognitive transmission. In Section IV, we propose a cognitive relay transmission scheme along with its performance analysis. Numerical results are also presented in this section. Finally, we make some concluding remarks in Section V.

\section{System Model}

Consider a cognitive radio network where a cognitive source (CS) is sending data to a cognitive destination (CD) over a spectrum hole unoccupied by a primary user (PU), as shown in Fig. 1 (a). Specifically, if CS detects an idle licensed frequency channel (that is not occupied currently by PU), it will use this channel for its data transmission (secondary data transmission); otherwise, CS will continue detecting the licensed frequency band to seek an available transmission opportunity. From Fig. 1, one can see that the whole cognitive transmission process is divided into two phases: 1) hole detection of the licensed band and 2) data transmission from CS to CD. The allocation of time durations between the two phases is depicted in Fig. 1 (b), where the detection phase and the transmission phase occupy $\alpha$ and 1- $\alpha$ fractions, respectively, of one time slot, and $\alpha$ is referred to as spectrum sensing overhead that can be varied to optimize the system performance. Notice that such a cognitive transmission protocol will be extended to a cognitive relay network in Section IV.

In Fig. 1 (a), each transmission link between any two nodes is modeled as a Rayleigh fading process and, moreover, the fading channel is considered as constant 
during one time slot. The transmit power of PU and CS are $P_{p}$ and $P_{s}$, respectively. For notational convenience, let $H_{p}(k, 1)$ and $H_{p}(k, 2)$ denote whether or not the licensed band is occupied by PU in the first and second phases, respectively, of time slot $k$, i.e, $H_{p}(k, 1)=H_{p}(k, 2)=H_{0}$ represents the band being unoccupied by $\mathrm{PU}$ and, otherwise, $H_{p}(k, 1)=H_{p}(k, 2)=H_{1}$. A Bernoulli distribution with parameter $\mathrm{P}_{\mathrm{a}}$ is used to model the random variable $H_{p}(k, 1)$, i.e., $\operatorname{Pr}\left(H_{p}(k, 1)=H_{0}\right)=\mathrm{P}_{\mathrm{a}}$ and $\operatorname{Pr}\left(H_{p}(k, 1)=H_{1}\right)=1-\mathrm{P}_{\mathrm{a}}$. Moreover, the transition between $H_{p}(k, 1)$ and $H_{p}(k, 2)$ is modeled as a Markov chain with parameter $1-\exp [-(1-\alpha) \lambda]$, i.e., $\quad \operatorname{Pr}\left(H_{p}(k, 2)=H_{1} \mid H_{p}(k, 1)=H_{0}\right)=$ $\operatorname{Pr}\left(H_{p}(k, 2)=H_{0} \mid H_{p}(k, 1)=H_{1}\right)=1-\exp [-(1-\alpha) \lambda]$ and $\quad \operatorname{Pr}\left(H_{p}(k, 2)=H_{0} \mid H_{p}(k, 1)=H_{0}\right)=$ $\operatorname{Pr}\left(H_{p}(k, 2)=H_{1} \mid H_{p}(k, 1)=H_{1}\right) \quad=\quad \exp [-(1-\alpha) \lambda]$, where $\lambda$ is the characteristic parameter. Thus, the signal detected by CS from PU can be expressed as

$$
y_{s}(k)=h_{p s}(k) \sqrt{P_{p}} \theta(k, 1)+n_{s}(k)
$$

where $h_{p s}(k)$ is the fading coefficient of the channel from PU to CS at time slot $k, n_{s}(k)$ is AWGN with zero mean and the power spectral density $N_{0}$, and $\theta(k, 1)$ is defined as

$$
\theta(k, 1)= \begin{cases}0, & H_{p}(k, 1)=H_{0} \\ x_{p}(k, 1), & H_{p}(k, 1)=H_{1}\end{cases}
$$

where $x_{p}(k, 1)$ is the transmission signal of $\mathrm{PU}$ during the first phase (namely hole detection phase) of time slot $k$. Based on (1), CS will make a decision $\hat{H}_{s}(k)$ on whether the licensed band is occupied by PU. Specifically, $\hat{H}_{s}(k)=H_{0}$ considers that the band is available for secondary transmissions; $\hat{H}_{s}(k)=H_{1}$ considers the band is unavailable. Therefore, the signal received at $\mathrm{CD}$ from $\mathrm{CS}$ can be written as

$$
y_{d}(k)=h_{s d}(k) \sqrt{P_{s}} \beta(k)+h_{p d}(k) \sqrt{P_{p}} \theta(k, 2)+n_{d}(k)
$$

where $h_{s d}(k)$ and $h_{p d}(k)$ are the fading coefficients of the channel from CS to CD and that from PU to CD, respectively. In addition, $\beta(k)$ and $\theta(k, 2)$ are defined as follows,

$$
\beta(k)= \begin{cases}x_{s}(k), & \hat{H}_{s}(k)=H_{0} \\ 0, & \hat{H}_{s}(k)=H_{1}\end{cases}
$$

and

$$
\theta(k, 2)= \begin{cases}0, & H_{p}(k, 2)=H_{0} \\ x_{p}(k, 2), & H_{p}(k, 2)=H_{1}\end{cases}
$$

where $x_{s}(k)$ and $x_{p}(k, 2)$ are the transmission signals of CS at time slot $k$ and that of PU at the second phase of time slot $k$, respectively.

\section{Outage Analysis of Cognitive Transmissions IN RAYLEIGH FADING CHANNELS}

In this section, we start with the outage analysis of cognitive transmissions and then conduct the numerical evaluations to show the impact of spectrum sensing overhead.

\section{A. Outage Probability Analysis}

As is known [31], [16], [19], an outage event occurs when the channel capacity falls below the data rate $R$. Following (2), the outage probability of cognitive transmissions is given by (3) at the top of the following page, where the coefficient $1-\alpha$ is due to the fact that only $1-\alpha$ fraction of a time slot is utilized for the CS's data transmission phase. Considering conditional probabilities, (3) can be expanded as (4) at the top of the following page, where $\Lambda=\left[2^{R /(1-\alpha)}-1\right] / \gamma_{s}$, $\gamma_{s}=P_{s} / N_{0}$ and $\gamma_{p}=P_{p} / N_{0}$. For notational convenience, let $\mathrm{P}_{\mathrm{a}}=\operatorname{Pr}\left(H_{p}(k, 1)=H_{0}\right)$ be the probability that there is a hole, $\mathrm{P}_{\mathrm{f}}=\operatorname{Pr}\left(\hat{H}_{s}(k)=H_{1} \mid H_{p}(k, 1)=H_{0}\right)$ and $\mathrm{P}_{\mathrm{d}}=$ $\operatorname{Pr}\left(\hat{H}_{s}(k)=H_{1} \mid H_{p}(k, 1)=H_{1}\right)$ be the probability of false alarm and the probability of detection of the primary-userpresence, respectively. Accordingly, (4) can be rewritten as (5) at the top of the following page. Note that there are several approaches available for the spectrum hole detection, such as energy detector, matched filter detector and cyclostationary feature detector as shown in [5] - [13]. Throughout this paper, we consider the use of an energy detector for the spectrum sensing performance analysis. Accordingly, considering an energy detection method and following (1), the output statistic of energy detector at time slot $k$ is given by

$$
T(y)=\frac{1}{N} \sum_{n=1}^{N}\left|y_{s}(k, n)\right|^{2}
$$

where $N=\alpha T f_{s}$ is the number of samples, $T$ and $f_{s}$ are the time slot length and sample frequency, respectively. From (6), the probability of false alarm and the probability of detection, $\mathrm{P}_{\mathrm{f}}(k)$ and $\mathrm{P}_{\mathrm{d}}(k)$, of the primary-user-presence at time slot $k$ are calculated as

$$
\begin{aligned}
\mathrm{P}_{\mathrm{f}}(k) & =\operatorname{Pr}\left(\hat{H}_{s}(k)=H_{1} \mid H_{p}(k, 1)=H_{0}\right) \\
& =\operatorname{Pr}\left\{T(y)>\delta \mid H_{p}(k, 1)=H_{0}\right\}
\end{aligned}
$$

and

$$
\begin{aligned}
\mathrm{P}_{\mathrm{d}}(k) & =\operatorname{Pr}\left(\hat{H}_{s}(k)=H_{1} \mid H_{p}(k, 1)=H_{1}\right) \\
& =\operatorname{Pr}\left\{T(y)>\delta \mid H_{p}(k, 1)=H_{1}\right\}
\end{aligned}
$$

where $\delta$ is the energy detection threshold. Using the results of Appendix A, we obtain

$$
\begin{aligned}
\mathrm{P}_{\text {out }}= & \mathrm{P}_{\mathrm{a}} \\
+ & \exp [-(1-\alpha) \lambda]\left(1-\mathrm{P}_{\mathrm{f}}\right)\left[1-\exp \left(-\frac{\Lambda}{\sigma_{s d}^{2}}\right)\right] \\
+ & \mathrm{P}_{\mathrm{a}}[1-\exp (-(1-\alpha) \lambda)]\left(1-\mathrm{P}_{\mathrm{f}}\right) \\
& \times\left[1-\frac{\sigma_{s d}^{2}}{\sigma_{p d}^{2} \gamma_{p} \Lambda+\sigma_{s d}^{2}} \exp \left(-\frac{\Lambda}{\sigma_{s d}^{2}}\right)\right] \\
+ & \left(1-\mathrm{P}_{\mathrm{a}}\right)[1-\exp (-(1-\alpha) \lambda)]\left(1-\mathrm{P}_{\mathrm{d}}\right) \\
& \times\left[1-\exp \left(-\frac{\Lambda}{\sigma_{s d}^{2}}\right)\right] \\
+ & \left(1-\mathrm{P}_{\mathrm{a}}\right) \exp [-(1-\alpha) \lambda]\left(1-\mathrm{P}_{\mathrm{d}}\right) \\
& \times\left[1-\frac{\sigma_{s d}^{2}}{\sigma_{p d}^{2} \gamma_{p} \Lambda+\sigma_{s d}^{2}} \exp \left(-\frac{\Lambda}{\sigma_{s d}^{2}}\right)\right] \\
+ & \mathrm{P}_{\mathrm{a}} \mathrm{P}_{\mathrm{f}}+\left(1-\mathrm{P}_{\mathrm{a}}\right) \mathrm{P}_{\mathrm{d}}
\end{aligned}
$$




$$
\begin{aligned}
& \mathrm{P}_{\text {out }}=\operatorname{Pr}\left\{(1-\alpha) \log \left(1+\frac{\left|h_{s d}(k)\right|^{2} P_{s}|\beta(k)|^{2}}{\left|h_{p d}(k)\right|^{2} P_{p}|\theta(k, 2)|^{2}+N_{0}}\right)<R\right\} \\
& \mathrm{P}_{\text {out }}= \operatorname{Pr}\left(\hat{H}_{s}(k)=H_{0}, H_{p}(k, 1)=H_{0}, H_{p}(k, 2)=H_{0}\right) \operatorname{Pr}\left\{\left|h_{s d}(k)\right|^{2}<\Lambda\right\} \\
&+ \operatorname{Pr}\left(\hat{H}_{s}(k)=H_{0}, H_{p}(k, 1)=H_{0}, H_{p}(k, 2)=H_{1}\right) \operatorname{Pr}\left\{\left|h_{s d}(k)\right|^{2}-\left|h_{p d}(k)\right|^{2} \gamma_{p} \Lambda<\Lambda\right\} \\
&+ \operatorname{Pr}\left(\hat{H}_{s}(k)=H_{0}, H_{p}(k, 1)=H_{1}, H_{p}(k, 2)=H_{0}\right) \operatorname{Pr}\left\{\left|h_{s d}(k)\right|^{2}<\Lambda\right\} \\
&+ \operatorname{Pr}\left(\hat{H}_{s}(k)=H_{0}, H_{p}(k, 1)=H_{1}, H_{p}(k, 2)=H_{1}\right) \operatorname{Pr}\left\{\left|h_{s d}(k)\right|^{2}-\left|h_{p d}(k)\right|^{2} \gamma_{p} \Lambda<\Lambda\right\} \\
&+ \operatorname{Pr}\left(\hat{H}_{s}(k)=H_{1}\right) \\
& \\
& \mathrm{P}_{\text {out }}=\mathrm{P}_{\mathrm{a}} \exp [-(1-\alpha) \lambda]\left(1-\mathrm{P}_{\mathrm{f}}\right) \operatorname{Pr}\left\{\left|h_{s d}(k)\right|^{2}<\Lambda\right\} \\
&+\mathrm{P}_{\mathrm{a}}[1-\exp (-(1-\alpha) \lambda)]\left(1-\mathrm{P}_{\mathrm{f}}\right) \operatorname{Pr}\left\{\left|h_{s d}(k)\right|^{2}-\left|h_{p d}(k)\right|^{2} \gamma_{p} \Lambda<\Lambda\right\} \\
&+\left(1-\mathrm{P}_{\mathrm{a}}\right)[1-\exp (-(1-\alpha) \lambda)]\left(1-\mathrm{P}_{\mathrm{d}}\right) \operatorname{Pr}\left\{\left|h_{s d}(k)\right|^{2}<\Lambda\right\} \\
&+\left(1-\mathrm{P}_{\mathrm{a}}\right) \exp [-(1-\alpha) \lambda]\left(1-\mathrm{P}_{\mathrm{d}}\right) \operatorname{Pr}\left\{\left|h_{s d}(k)\right|^{2}-\left|h_{p d}(k)\right|^{2} \gamma_{p} \Lambda<\Lambda\right\} \\
&+\mathrm{P}_{\mathrm{a}} \mathrm{P}_{\mathrm{f}}+\left(1-\mathrm{P}_{\mathrm{a}}\right) \mathrm{P}_{\mathrm{d}}
\end{aligned}
$$

where the false alarm probability $\mathrm{P}_{\mathrm{f}}$ is constrained to the detection probability $\mathrm{P}_{\mathrm{d}}$ as given below

$\mathrm{P}_{\mathrm{f}}= \begin{cases}\mathrm{P}_{\mathrm{d}}, & \mathrm{P}_{\mathrm{d}}=Q(-\sqrt{N}) \\ \mathrm{P}_{\mathrm{d}}-Q\left(Q^{-1}\left(\mathrm{P}_{\mathrm{d}}\right)+\frac{1}{\sigma_{p s}^{2} \kappa}\right) \exp (\xi), & \text { otherwise }\end{cases}$

where $\kappa=\gamma_{p} Q^{-1}\left(\mathrm{P}_{\mathrm{d}}\right)+\sqrt{N} \gamma_{p}, \xi=\frac{Q^{-1}\left(\mathrm{P}_{\mathrm{d}}\right)}{\sigma_{\mathrm{ps}}^{2} \kappa}+\frac{1}{2 \sigma_{\mathrm{ps}}^{4} \kappa^{2}}$, and the number of samples $N$ should satisfy $N \geq\left[Q^{-1}\left(\mathrm{P}_{\mathrm{d}}\right)\right]^{2}$. As can be observed from (9), the derived closed-from expression of outage probability accounts for both the probability of no available channel detected in the spectrum hole detection phase and the probability of channel outage occurred in the subsequent data transmission phase, and thus we call it overall outage probability. Following (9) and letting $\gamma_{s} \rightarrow+\infty$, we are able to obtain an overall outage probability floor

$$
\mathrm{P}_{\text {out }, \text { floor }}=\mathrm{P}_{\mathrm{a}} \mathrm{P}_{\mathrm{f}}+\left(1-\mathrm{P}_{\mathrm{a}}\right) \mathrm{P}_{\mathrm{d}}
$$

which can also be explained as follows. Once CS detects a spectrum hole (no matter it is a correct or a false detection) in the first phase, a channel outage would not occur in the subsequent data transmission phase due to $\gamma_{s} \rightarrow+\infty$. In other words, when $\gamma_{s} \rightarrow+\infty$, the outage occurs only when CS detects the presence of PU (i.e., no hole is detected) no matter whether PU really presents or not. Combining (9) and (10), one can see that the overall outage probability of the cognitive transmission is a function of $\left\{\mathrm{P}_{\mathrm{a}}, \mathrm{P}_{\mathrm{d}}, \mathrm{P}_{\mathrm{f}}, R\right.$, $\left.\sigma_{p s}^{2}, \sigma_{p d}^{2}, \sigma_{s d}^{2}, \gamma_{s}, \gamma_{p}, \alpha\right\}$, where the data rate $R$ may often be set by the system in accordance with the QoS requirement and the network environment, and the spectrum sensing overhead $\alpha$ is a parameter that can be adapted to optimize the system performance. To obtain a general expression for an optimal $\alpha^{*}$ as a function of the other parameters is very complicated and infeasible. Here, we consider an asymptotic outage probability analysis to decide the optimal value $\alpha^{*}$. Considering the case of $\mathrm{P}_{\mathrm{a}}=1$ and $\lambda=0$ and letting $\gamma_{s} \rightarrow \infty$, we can obtain from (9) using the Taylor approximation as

$$
\mathrm{P}_{\text {out }} \cong\left(1-\mathrm{P}_{\mathrm{f}}\right) \frac{\Lambda}{\sigma_{s d}^{2}}+\mathrm{P}_{\mathrm{f}}
$$

Letting $\gamma_{p} \rightarrow \infty$, we can similarly apply the Taylor approximation to (10) and obtain

$$
\begin{aligned}
\mathrm{P}_{\mathrm{f}} & \cong \mathrm{P}_{\mathrm{d}}-\mathrm{P}_{\mathrm{d}}\left(1+\frac{Q^{-1}\left(\mathrm{P}_{\mathrm{d}}\right)}{\left[Q^{-1}\left(\mathrm{P}_{\mathrm{d}}\right)+\sqrt{N}\right] \sigma_{p s}^{2}} \cdot \frac{1}{\gamma_{p}}\right) \\
& =-\frac{\mathrm{P}_{\mathrm{d}} Q^{-1}\left(\mathrm{P}_{\mathrm{d}}\right)}{\left[Q^{-1}\left(\mathrm{P}_{\mathrm{d}}\right)+\sqrt{\alpha T f_{s}}\right] \sigma_{p s}^{2}} \cdot \frac{1}{\gamma_{p}}
\end{aligned}
$$

In obtaining the first equation of (13), we have ignored the term $1 /\left(2 \sigma_{p s}^{4} \kappa^{2}\right)$, since it is a higher-order infinitesimal compared to the term $Q^{-1}\left(\mathrm{P}_{\mathrm{d}}\right) /\left(\sigma_{p s}^{2} \kappa\right)$ for $\gamma_{p} \rightarrow \infty$. Differentiating (12) with respective to the spectrum sensing overhead $\alpha$ yields

$$
\begin{aligned}
\frac{\partial \mathrm{P}_{\mathrm{out}}}{\partial \alpha} & =\left(1-\mathrm{P}_{\mathrm{f}}\right) \frac{\partial \Lambda}{\sigma_{s d}^{2} \partial \alpha}+\left(1-\frac{\Lambda}{\sigma_{s d}^{2}}\right) \frac{\partial \mathrm{P}_{\mathrm{f}}}{\partial \alpha} \\
& \cong \frac{\partial \Lambda}{\sigma_{s d}^{2} \partial \alpha}+\frac{\partial \mathrm{P}_{\mathrm{f}}}{\partial \alpha}
\end{aligned}
$$

where the second equation is obtained due to the fact that $\mathrm{P}_{\mathrm{f}}$ and $\Lambda$ are infinitesimal when $\gamma_{p}, \gamma_{s} \rightarrow \infty$. Meanwhile, $\partial \mathrm{P}_{\mathrm{f}} / \partial \alpha$ and $\partial \Lambda / \partial \alpha$ are given by

$$
\frac{\partial \mathrm{P}_{\mathrm{f}}}{\partial \alpha}=\frac{\mathrm{P}_{\mathrm{d}} Q^{-1}\left(\mathrm{P}_{\mathrm{d}}\right) \sqrt{T f_{s}}}{2 \gamma_{p} \sigma_{p s}^{2} \sqrt{\alpha}\left[Q^{-1}\left(\mathrm{P}_{\mathrm{d}}\right)+\sqrt{\alpha T f_{s}}\right]^{2}}
$$

and

$$
\frac{\partial \Lambda}{\partial \alpha}=\frac{R 2^{R /(1-\alpha)} \ln 2}{(1-a)^{2} \gamma_{s}}
$$

By substituting $\partial \mathrm{P}_{\mathrm{f}} / \partial \alpha$ and $\partial \Lambda / \partial \alpha$ from (15) and (16) into (14), the optimal spectrum sensing overhead should satisfy the following equation

$$
2^{R /\left(1-\alpha^{*}\right)} \sqrt{\alpha^{*}}\left[\sqrt{\alpha^{*} T f_{s}}+Q^{-1}\left(\mathrm{P}_{\mathrm{d}}\right)\right]^{2} \xi+\left(1-\alpha^{*}\right)^{2} \zeta=0
$$


TABLE I

LIST OF THE OPTIMAL SPECTRUM SENSING OVERHEAD $\alpha^{*}$ UNDER THE DIFFERENT PRIMARY AND SECONDARY TRANSMIT SIGNAL-TO-NOISE RATIOS $\left(\gamma_{s}, \gamma_{p}\right)$.

\begin{tabular}{|c|c|c|c|}
\hline$\left(\gamma_{s}, \gamma_{p}\right)$ & $\alpha^{*}$ & $\left(\gamma_{s}, \gamma_{p}\right)$ & $\alpha^{*}$ \\
\hline$(20,30)$ & 0.02219 & $(30,20)$ & 0.21934 \\
\hline$(20,35)$ & 0.01286 & $(35,20)$ & 0.33492 \\
$(20,40)$ & 0.00796 & $(40,20)$ & 0.45913 \\
\hline
\end{tabular}

wherein $\xi$ and $\zeta$ are given by

$$
\xi=\sigma_{p s}^{2} \gamma_{p} R \ln 4 ; \quad \zeta=\sigma_{s d}^{2} \gamma_{s} \sqrt{T f_{s}} \mathrm{P}_{\mathrm{d}} Q^{-1}\left(\mathrm{P}_{\mathrm{d}}\right)
$$

An optimal spectrum sensing overhead $\alpha^{*}$ can be easily determined by using (17). In Table 1, we show the optimal sensing overhead under different values of the primary and secondary transmit powers $\left(\gamma_{s}, \gamma_{p}\right)$ with $\mathrm{P}_{\mathrm{d}}=0.99, \sigma_{p s}^{2}=\sigma_{s d}^{2}=1$, $R=1 \mathrm{bit} / \mathrm{s} / \mathrm{Hz}, T=25 \mathrm{~ms}$ and $f_{s}=100 \mathrm{kHz}$. As shown in the first two columns of Table 1 , as the primary transmit power $\gamma_{p}$ increases, the optimal spectrum sensing overhead $\alpha^{*}$ decreases, which is due to the fact that less time duration is required for the spectrum hole detection phase with an increasing primary transmit power. From the last two columns of Table 1, one can also see that the optimal spectrum sensing overhead increases when the secondary transmit power $\gamma_{s}$ increases from $\gamma_{s}=30 \mathrm{~dB}$ to $\gamma_{s}=40 \mathrm{~dB}$. This is because that as the secondary transmit power increases, less time is needed for the data transmission phase, thus resulting in a longer time duration available for the spectrum sensing phase.

\section{B. Numerical Results and Analysis}

In this subsection, we focus on the numerical evaluations to show the impact of spectrum sensing overhead on the overall outage probability performance. Notice that the primary user would be interfered by the cognitive user when CS does not detect the presence of PU given that PU is active. Therefore, the probability of detection of the primary-user-presence $\mathrm{P}_{\mathrm{d}}$ shall be set to a required threshold by the cognitive system to guarantee PU's QoS. Throughout this paper, we will use $\mathrm{P}_{\mathrm{d}}=0.99$ for the numerical evaluations.

Fig. 2 depicts the probability of detection versus the probability of false alarm using (10). It is shown from Fig. 2 that, under a required detection probability, as the spectrum sensing overhead $\alpha$ increases, the false alarm probability decreases, i.e., CS can detect the hole of licensed band more accurately. This result is easy to understand since increasing $\alpha$ indicates the enhancement of the signal energy received at CS from PU, which thus leads to the detection performance improvement.

Fig. 3 plots (9) as a function of the transmit SNR at CS $\gamma_{s}$ for different spectrum sensing overhead values. The corresponding outage probability floors for the three cases (i.e., $\alpha=0.1, \alpha=0.2$ and $\alpha=0.5$ ) are plotted using (8). Also, the simulated results are provided in this figure by using a typical link-level simulation. As shown in Fig. 3, the curves

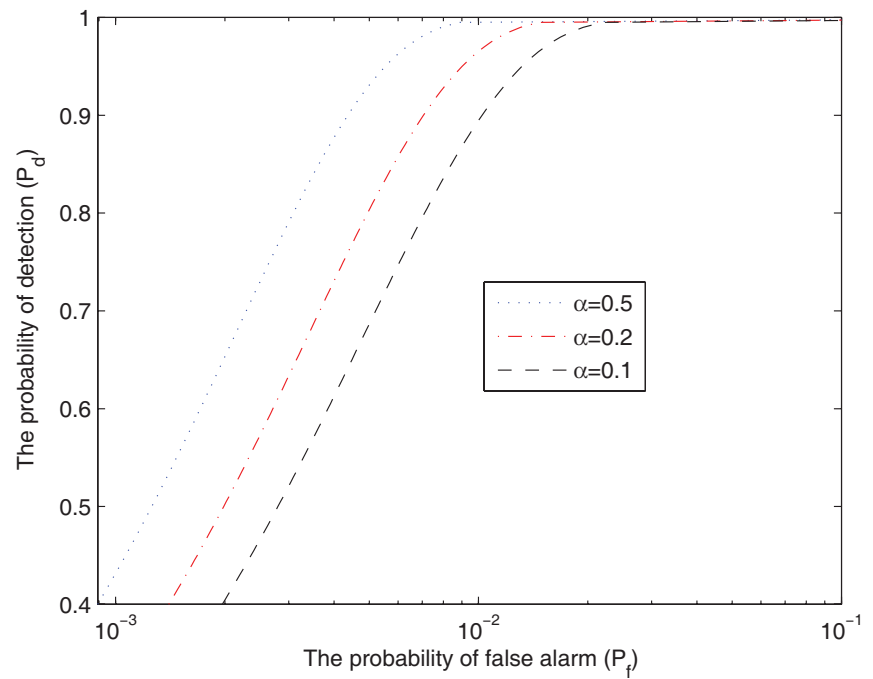

Fig. 2. The probability of detection versus the probability of false alarm for different values of the spectrum sensing overhead $\alpha$ with $\gamma_{p}=10 \mathrm{~dB}$, $T=20 \mathrm{~ms}, f_{s}=100 \mathrm{kHz}$, and $\sigma_{p s}^{2}=1$.

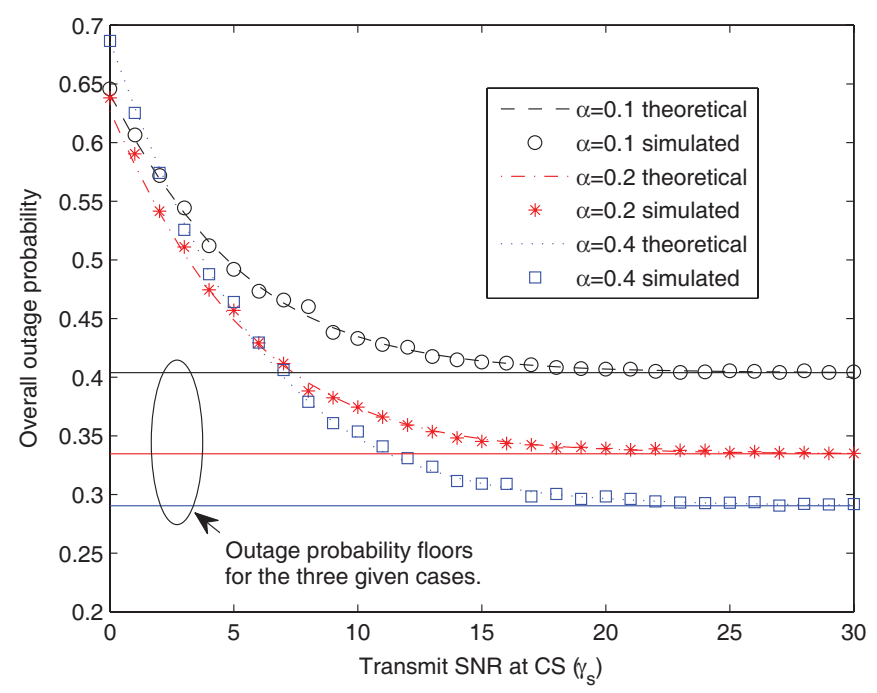

Fig. 3. Overall outage probability versus the transmit SNR at CS $\gamma_{s}$ for different values of the spectrum sensing overhead $\alpha$ with $\mathrm{P}_{\mathrm{a}}=0.8, \lambda=0.1$, $\mathrm{P}_{\mathrm{d}}=0.99, R=0.5 \mathrm{~b} / \mathrm{s} / \mathrm{Hz}, \gamma_{p}=5 \mathrm{~dB}, T=20 \mathrm{~ms}, f_{s}=25 \mathrm{kHz}$, $\sigma_{p s}^{2}=\sigma_{p d}^{2}=0.5$, and $\sigma_{s d}^{2}=1$.

of the overall outage probability converge to the corresponding floors in high $\gamma_{s}$ regions. The occurrence of outage floor is due to the fact that when the transmit SNR $\gamma_{s}$ is very high, the overall outage probability is dominated by the probability of no spectrum hole detected that is independent from $\gamma_{s}$. Moreover, the floors observed are reduced with the increase of the overhead $\alpha$, which is resulted from the improvement of the detection probability of spectrum holes as the overhead $\alpha$ increases. In addition, the simulation results match the analytical results very well.

In Fig. 4, we show the overall outage probability versus the spectrum sensing overhead $\alpha$ for different values of the data rate, where the performance curves plotted correspond to $R=0.4 \mathrm{~b} / \mathrm{s} / \mathrm{Hz}$ and $R=0.8 \mathrm{~b} / \mathrm{s} / \mathrm{Hz}$, respectively. All the curves plotted in Fig. 4 demonstrate that there always exists an optimal spectrum sensing overhead $\alpha^{*}$, i.e., a minimized 


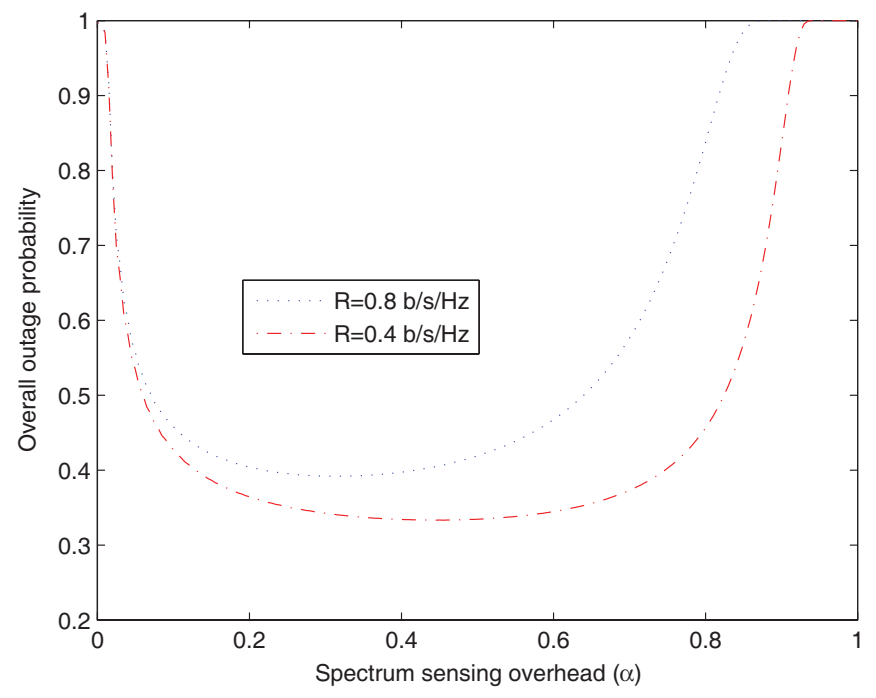

Fig. 4. Overall outage probability versus the spectrum sensing overhead $\alpha$ for different values of the data rate $R$ with $\mathrm{P}_{\mathrm{a}}=0.8, \lambda=0.1, \mathrm{P}_{\mathrm{d}}=0.99$, $\gamma_{s}=10 \mathrm{~dB}, \gamma_{p}=5 \mathrm{~dB}, T=20 \mathrm{~ms}, f_{s}=25 \mathrm{kHz}, \sigma_{p s}^{2}=\sigma_{p d}^{2}=0.5$, and $\sigma_{s d}^{2}=1$.

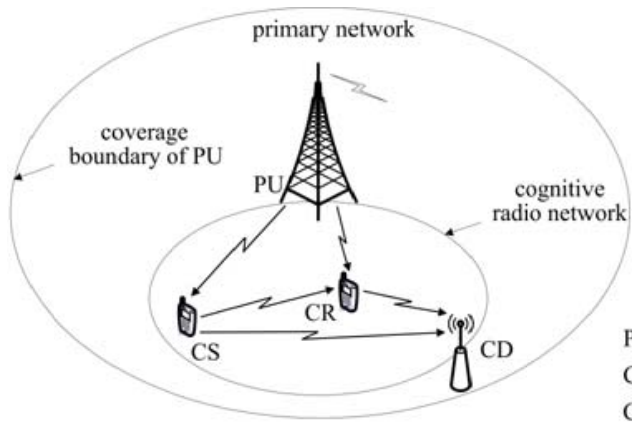

PU: Primary User CS: Cognitive Source CR: Cognitive Relay

(a) $\mathrm{CD}$ : Cognitive Destination

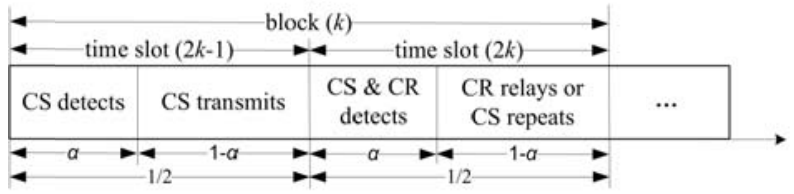

(b)

Fig. 5. (a) Coexistence of a primary wireless network and a cognitive relay network; (b) The allocation of time durations: Hole detection versus data transmission.

outage probability can be achieved through a tradeoff in determining the time durations for the hole detection and the data transmission phases. It is noted that for any given parameter set, an optimal spectrum sensing overhead $\alpha^{*}$ can be determined by minimizing the overall outage probability as given by (9).

\section{Combined Cognitive Relay and Cognitive TRANSMISSION}

In this section, we consider a cognitive relay network as shown in Fig. 5 (a), where a cognitive relay (CR) assists CS for the data transmission. Fig. 5 (b) illustrates the proposed cognitive relay transmission process, which is described in detail as follows. At the first phase of time slot $2 k-1$, CS detects the status of the licensed band. If a spectrum hole is detected, CS transmits its signal to both $\mathrm{CR}$ and $\mathrm{CD}$ during the second phase of the same time slot. Next, CR decodes its received signal from CS during the first phase of time slot $2 k$ and, meanwhile, detects whether or not there is a spectrum hole. If the signal is decoded successfully and a hole is detected, CR will notify CS and forward its decoded signal to $\mathrm{CD}$ during the next phase. Otherwise, CS will determine whether or not to repeat its signal transmission directly to $\mathrm{CD}$ depending on its hole detection result at the second phase of time slot $2 k$.

As shown in Fig. 6, we present a block diagram of the general framework for implementation of the proposed cognitive relay transmission scheme. An original bit stream are processed in FED (forward error-detecting, e.g., a cyclic redundancy check (CRC) code) and FEC (forward errorcorrecting, e.g., Turbo code) encoders at time slot $2 k-1$, giving a encoded bit stream that would be transmitted to both $\mathrm{CR}$ and $\mathrm{CD}$ when a spectrum hole is detected through the hole detection module. At the next time slot $2 k$, CR performs FEC decoding and FED checking on the received bits. If the FED check is successful and a spectrum hole is detected, CR would then transmit the decoded bits to CD. Otherwise, CS would determine whether or not to repeat the transmission of the original bits depending on its hole detection result at time slot $2 k$. Finally, CD combines the two copies of received signals by using maximum ratio combining (MRC) method, and gives an estimated bit stream of the original bits after a FEC decoder.

Let $H_{p}(2 k-1)$ and $H_{p}(2 k)$ denote the statuses of licensed band at time slots $2 k-1$ and $2 k$, respectively. Similarly, let $\hat{H}_{s}(2 k-1)$ and $\hat{H}_{r}(2 k)$ be the decision results made by $\mathrm{CS}$ and $\mathrm{CR}$, respectively, as to whether there is a spectrum hole. For simplicity, we assume here that the primary traffic status does not change during one time slot. Notice that following the procedures as stated in Section III, the analysis can be extended with additional primary traffic states to the scenario where the primary traffic status may change between the spectrum hole detection and data transmission phases, for which similar performance characteristics can be obtained. Here, we use a Bernoulli distribution with parameter $\mathrm{P}_{\mathrm{a}}$ to model the random variable $H_{p}(2 k-1)$, i.e., $\operatorname{Pr}\left(H_{p}(2 k-1)=H_{0}\right)=\mathrm{P}_{\mathrm{a}}$ and $\operatorname{Pr}\left(H_{p}(2 k-1)=H_{1}\right)=1-\mathrm{P}_{\mathrm{a}}$. Moreover, a Markov chain with a transition probability $\mathrm{P}_{\mathrm{t}}=1-\exp (-\lambda)$ is used to model the process of primary traffic arrival/departure in two adjacent time slots, i.e., $\quad \operatorname{Pr}\left(H_{p}(2 k)=H_{1} \mid H_{p}(2 k-1)=H_{0}\right) \quad=$ $\operatorname{Pr}\left(H_{p}(2 k)=H_{0} \mid H_{p}(2 k-1)=H_{1}\right)=\mathrm{P}_{\mathrm{t}}$ and $\quad \operatorname{Pr}\left(H_{p}(2 k)=H_{0} \mid H_{p}(2 k-1)=H_{0}\right) \quad=$ $\operatorname{Pr}\left(H_{p}(2 k)=H_{1} \mid H_{p}(2 k-1)=H_{1}\right)=1-\mathrm{P}_{\mathrm{t}}$. Besides, each wireless link as depicted in Fig. 5 is modeled as a Rayleigh block fading channel, i.e., the channel fading is invariant during one whole block consisting of two time slots.

\section{A. Signal Modeling and Outage Analysis}

Following [16], [19] and considering for practicability, we would adopt half-duplex relay to model and analyze the cognitive relay transmission scheme. At time slot $2 k-1$, the 


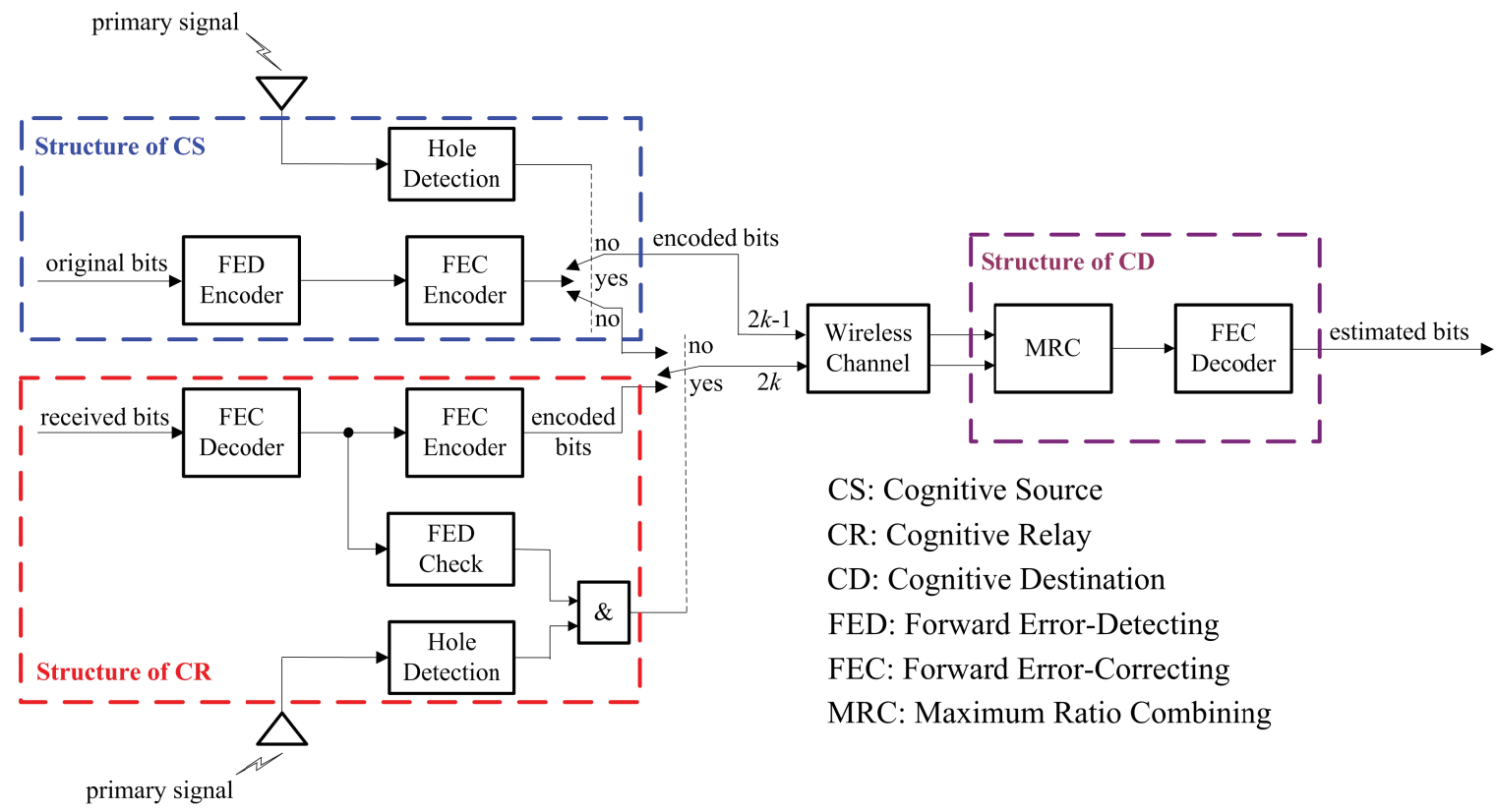

Fig. 6. A block diagram of the general framework for implementation of the proposed cognitive relay transmission scheme.

CS signals received at $\mathrm{CR}$ and $\mathrm{CD}$ can be expressed as

$$
\begin{aligned}
y_{r}(2 k-1)= & h_{s r}(k) \sqrt{P_{s}} \beta(2 k-1) \\
& +h_{p r}(k) \sqrt{P_{p}} \theta(2 k-1,2)+n_{r}(2 k-1)
\end{aligned}
$$

and

$$
\begin{aligned}
y_{d}(2 k-1)= & h_{s d}(k) \sqrt{P_{s}} \beta(2 k-1) \\
& +h_{p d}(k) \sqrt{P_{p}} \theta(2 k-1,2)+n_{d}(2 k-1)
\end{aligned}
$$

where

$$
\begin{aligned}
& \beta(2 k-1)= \begin{cases}x_{s}(k), & \hat{H}_{s}(2 k-1)=H_{0} \\
0, & \hat{H}_{s}(2 k-1)=H_{1}\end{cases} \\
& \theta(2 k-1,2)= \begin{cases}0, & H_{p}(2 k-1)=H_{0} \\
x_{p}(2 k-1,2), & H_{p}(2 k-1)=H_{1}\end{cases}
\end{aligned}
$$

where $x_{p}(2 k-1,2)$ is the transmit signal of $\mathrm{PU}$ at the second phase of time slot $2 k-1$. As depicted in Fig. 5 (b), there exist two data transmission cases (dependent on whether or not CR can decode successfully and detect a spectrum hole) at time slot $2 k$, which are denoted by $\Theta=1$ and $\Theta=2$, respectively. If $\mathrm{CR}$ succeeds in decoding its received signal and detects a spectrum hole at time slot $2 k$, it can be described as (21) at the top of the following page (in an information-theoretic sense [31]). where the factor $1 / 2$ in front of $\log (\cdot)$ is resulted from a half-duplex relay constraint [16]. Then, at the next phase of time slot $2 k, \mathrm{CR}$ would forward its correctly decoded result $\hat{x}_{s}(k)=x_{s}(k)$ to $\mathrm{CD}$. Thus, the signal received at $\mathrm{CD}$ can be written as

$$
\begin{aligned}
y_{d}(2 k, \Theta=1)= & h_{r d}(k) \sqrt{P_{s}} x_{s}(k) \\
& +h_{p d}(k) \sqrt{P_{p}} \theta(2 k, 2)+n_{d}(2 k)
\end{aligned}
$$

where

$$
\theta(2 k, 2)= \begin{cases}0, & H_{p}(2 k)=H_{0} \\ x_{p}(2 k, 2), & H_{p}(2 k)=H_{1}\end{cases}
$$

Note that $\beta(2 k-1)$ must be equal to $x_{s}(k)$ in the case of CR succeeding in decoding the received signal; otherwise, $\mathrm{CR}$ will not be able to decode successfully. By substituting this result into (20) and combining that with (22), CD achieves an enhanced signal version as given by (23), from which the signal-to-interference-and-noise ratio (SINR) is calculated as

$$
\begin{aligned}
& \operatorname{SINR}_{\mathrm{d}}(\Theta=1) \\
& =\frac{\left|h_{s d}(k)\right|^{2} \gamma_{s}+\left|h_{r d}(k)\right|^{2} \gamma_{s}}{\left|h_{p d}(k)\right|^{2} \gamma_{p}|\theta(2 k-1,2)|^{2}+\left|h_{p d}(k)\right|^{2} \gamma_{p}|\theta(2 k, 2)|^{2}+2}
\end{aligned}
$$

If CR fails to decode its received signal or to detect a spectrum hole at time slot $2 k$, such an event can be described as (25). In this case, CS, instead of CR, would determine whether or not to repeat the transmission of signal $x_{s}(k)$ to $\mathrm{CD}$ depending on its hole detection result at time slot $2 k$. In the case of $\Theta=2$, the signal received at $\mathrm{CD}$ from $\mathrm{CS}$ during the time slot $2 k$ can be written as

$$
\begin{aligned}
y_{d}(2 k, \Theta=2)= & h_{s d}(k) \sqrt{P_{s}} \beta(2 k)+h_{p d}(k) \sqrt{P_{p}} \theta(2 k, 2) \\
& +n_{d}(2 k)
\end{aligned}
$$

where $\beta(2 k)$ is defined as

$$
\beta(2 k)= \begin{cases}x_{s}(k), & \hat{H}_{s}(2 k)=H_{0} \\ 0, & \hat{H}_{s}(2 k)=H_{1}\end{cases}
$$

Combining (20) and (26) with MRC method, CD can achieve an enhanced signal version as given by

$$
\begin{aligned}
& \tilde{y}_{d}(2 k, \Theta=2)=h_{s d}(k) \sqrt{P_{s}} \beta(2 k-1)+h_{s d}(k) \sqrt{P_{s}} \beta(2 k) \\
& \quad+h_{p d}(k) \sqrt{P_{p}} \theta(2 k-1,2)+h_{p d}(k) \sqrt{P_{p}} \theta(2 k, 2) \\
& \quad+n_{d}(2 k-1)+n_{d}(2 k)
\end{aligned}
$$




$$
\begin{gathered}
\Theta=1: \frac{(1-\alpha)}{2} \log \left(1+\frac{\left|h_{s r}(k)\right|^{2} \gamma_{s}|\beta(2 k-1)|^{2}}{\left|h_{p r}(k)\right|^{2} \gamma_{p}|\theta(2 k-1,2)|^{2}+1}\right)>R \text { and } \hat{H}_{r}(2 k)=H_{0} \\
\tilde{y}_{d}(2 k, \Theta=1)=h_{s d}(k) \sqrt{P_{s}} x_{s}(k)+h_{r d}(k) \sqrt{P_{s}} x_{s}(k)+h_{p d}(k) \sqrt{P_{p}} \theta(2 k-1,2)+h_{p d}(k) \sqrt{P_{p}} \theta(2 k, 2)+n_{d}(2 k-1)+n_{d}(2 k) \\
\Theta=2: \frac{(1-\alpha)}{2} \log \left(1+\frac{\left|h_{s r}(k)\right|^{2} \gamma_{s}|\beta(2 k-1)|^{2}}{\left|h_{p r}(k)\right|^{2} \gamma_{p}|\theta(2 k-1,2)|^{2}+1}\right)<R \text { or } \hat{H}_{r}(2 k)=H_{1}
\end{gathered}
$$

from which the corresponding SINR can be calculated as

$$
\begin{aligned}
& \operatorname{SINR}_{\mathrm{d}}(\Theta=2) \\
& =\frac{\left|h_{s d}(k)\right|^{2} \gamma_{s}|\beta(2 k-1)|^{2}+\left|h_{s d}(k)\right|^{2} \gamma_{s}|\beta(2 k)|^{2}}{\left|h_{p d}(k)\right|^{2} \gamma_{p}|\theta(2 k-1,2)|^{2}+\left|h_{p d}(k)\right|^{2} \gamma_{p}|\theta(2 k, 2)|^{2}+2}
\end{aligned}
$$

Now, we have formulated the signal models for the cognitive relay transmission, based on which a detailed analysis of the overall outage probability is presented in the following, and also a performance comparison with the non-relay transmission is conducted to illustrate the advantage of the proposed cognitive relay scheme. According to the coding theorem [31], an outage event is deemed to occur when the channel capacity falls below the data rate $R$. Thus, we can calculate the overall outage probability of the cognitive relay transmission as

$$
\begin{aligned}
\mathrm{P}_{\text {out }, \text { relay }} & =\operatorname{Pr}\left\{\frac{1-\alpha}{2} \log \left(1+\operatorname{SINR}_{\mathrm{d}}\right)<R\right\} \\
& =\operatorname{Pr}\left\{\frac{1-\alpha}{2} \log \left(1+\operatorname{SINR}_{\mathrm{d}}(\Theta=1)\right)<R, \Theta=1\right\} \\
& +\operatorname{Pr}\left\{\frac{1-\alpha}{2} \log \left(1+\operatorname{SINR}_{\mathrm{d}}(\Theta=2)\right)<R, \Theta=2\right\} \\
& =\operatorname{Pr}\left\{\operatorname{SINR}_{\mathrm{d}}(\Theta=1)<\gamma_{s} \Delta, \Theta=1\right\} \\
& +\operatorname{Pr}\left\{\operatorname{SINR}_{\mathrm{d}}(\Theta=2)<\gamma_{s} \Delta, \Theta=2\right\}
\end{aligned}
$$

where $\Delta=\left[2^{2 R /(1-\alpha)}-1\right] / \gamma_{s}$. Using the results from Appendix B, we can obtain

$$
\mathrm{P}_{\text {out,relay }}=\mathrm{B} 2(\mathrm{a})+\mathrm{B} 2(\mathrm{~b})+\mathrm{B} 2(\mathrm{c})+\mathrm{B} 2(\mathrm{~d})+\mathrm{B} 12(\mathrm{a})-\mathrm{B} 12(\mathrm{~b})
$$

Closed-form expressions of B2(a), B2(b), B2(c), B2(d), $\mathrm{B} 12(\mathrm{a})$ and $\mathrm{B} 12(\mathrm{~b})$ are presented in Appendix B as given by (B.5), (B.7), (B.8), (B.9), (B.13) and (B.14), respectively. As can be seen from (30), obtaining a general expression for an optimal spectrum sensing overhead $\alpha$ as a function of the other parameters is very difficult. However, an optimal $\alpha$ value can be determined through numerical computation.

\section{B. Numerical Results and Analysis}

Fig. 7 shows the overall outage probability versus the transmit SNR $\gamma_{s}$ using (9) and (30), respectively, in which the simulated results are also given by employing a typical link-level simulation approach. From Fig. 7, one can see that in low SNR regions, the outage performance of the cognitive relay scheme performs worse than the non-relay transmission. This is because that out of consideration for practicability,

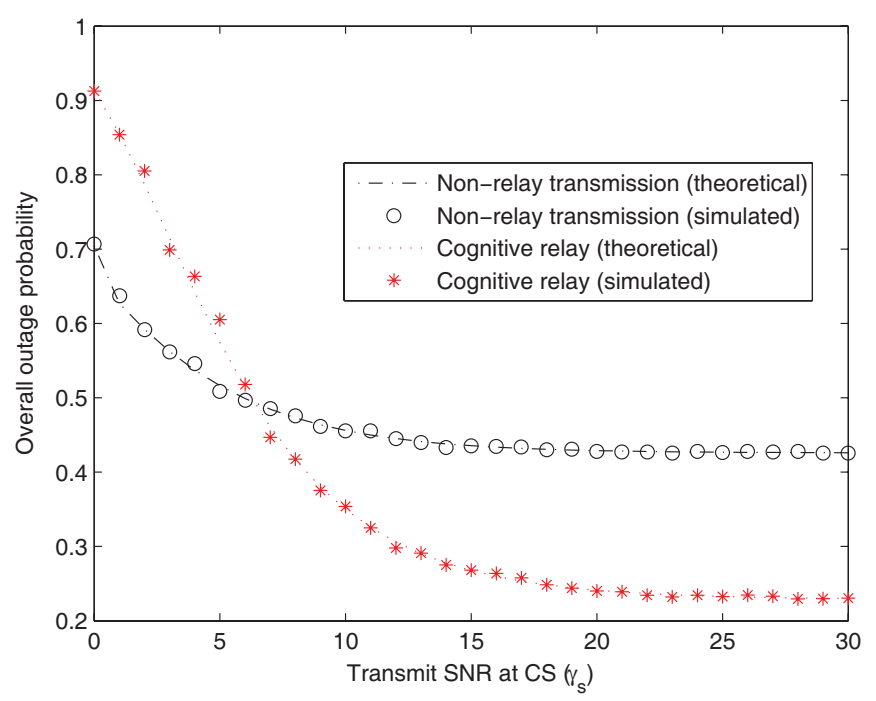

Fig. 7. Outage performance versus the transmit SNR at CS $\gamma_{s}$ of the nonrelay and the cognitive relay schemes with $\mathrm{P}_{\mathrm{a}}=0.8, \lambda=0.1, \mathrm{P}_{\mathrm{d}, \mathrm{s}}=$ $\mathrm{P}_{\mathrm{d}, \mathrm{r}}=0.99, \alpha=0.2, R=0.5 \mathrm{~b} / \mathrm{s} / \mathrm{Hz}, \gamma_{p}=5 \mathrm{~dB}, T=20 \mathrm{~ms}, f_{s}=$ $25 \mathrm{kHz}, \sigma_{p s}^{2}=\sigma_{p r}^{2}=\sigma_{p d}^{2}=0.2, \sigma_{s d}^{2}=1$, and $\sigma_{s r}^{2}=\sigma_{r d}^{2}=1.2$.

we adopt a half-duplex relay, rather than a full-duplex relay, which would degrade the system performance. However, in higher SNR regions, the benefits achieved overtake the costs and the performance of cognitive relay transmission outperforms the non-relay scheme. Note that in practical wireless communication systems, the transmit SNR shall be required to meet a satisfactory QoS and thus overly low transmit SNR is not practical. Accordingly, the advantage of the cognitive relay scheme is achieved in practical communication systems. It is worth mentioning that the overall outage probability floor of the cognitive relay scheme is reduced noticeably compared with the non-relay scheme, demonstrating the advantage of the cognitive relay scheme. Besides, one can see from Fig. 7 that the simulation results match the theoretical results well.

Fig. 8 illustrates the overall outage probability for the cognitive relay transmission by plotting (30) as a function of $\gamma_{s}$ for different spectrum sensing overhead $\alpha$ values. As shown in Fig. 8, the curves approach to their respective outage probability floors in high SNR regions and, also, the floors go down with the increase of spectrum sensing overhead $\alpha$. This is due to the fact that at high $\gamma_{s}$ values, the outage probability is dominantly dependent on the detection probability that can be improved as the overhead $\alpha$ increases. Besides, one can see from Fig. 8 that in lower SNR regions, the relationship 


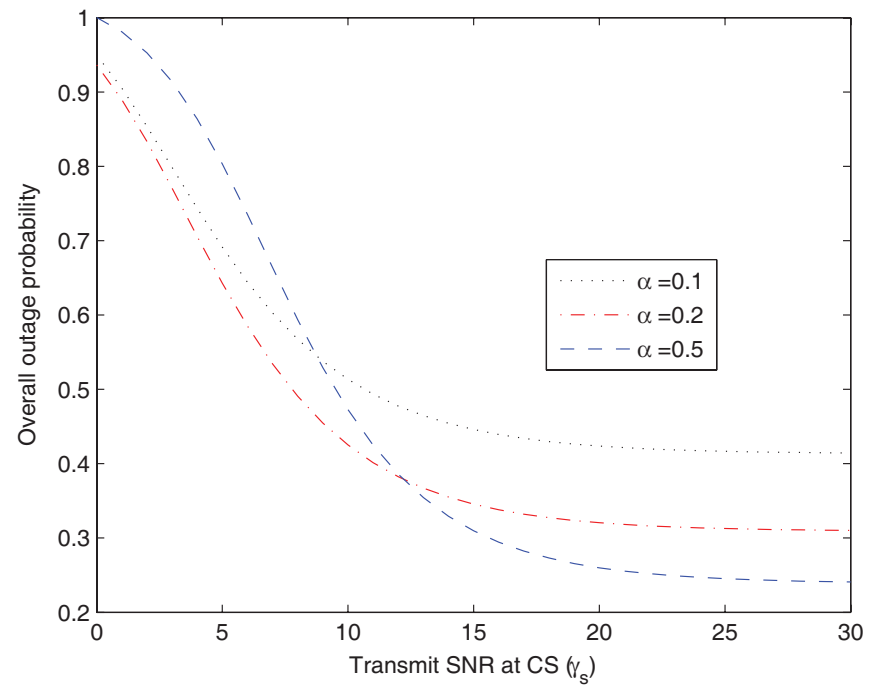

Fig. 8. Overall outage probability versus the transmit SNR at CS $\gamma_{s}$ for different values of the spectrum sensing overhead $\alpha$ with $\mathrm{P}_{\mathrm{a}}=0.8, \lambda=$ $0.1, \mathrm{P}_{\mathrm{d}, \mathrm{s}}=\mathrm{P}_{\mathrm{d}, \mathrm{r}}=0.99, R=0.5 \mathrm{~b} / \mathrm{s} / \mathrm{Hz}, \gamma_{p}=5 \mathrm{~dB}, T=20 \mathrm{~ms}$, $f_{s}=25 \mathrm{kHz}, \sigma_{p s}^{2}=\sigma_{p r}^{2}=\sigma_{p d}^{2}=0.2, \sigma_{s d}^{2}=1$, and $\sigma_{s r}^{2}=\sigma_{r d}^{2}=0.8$.

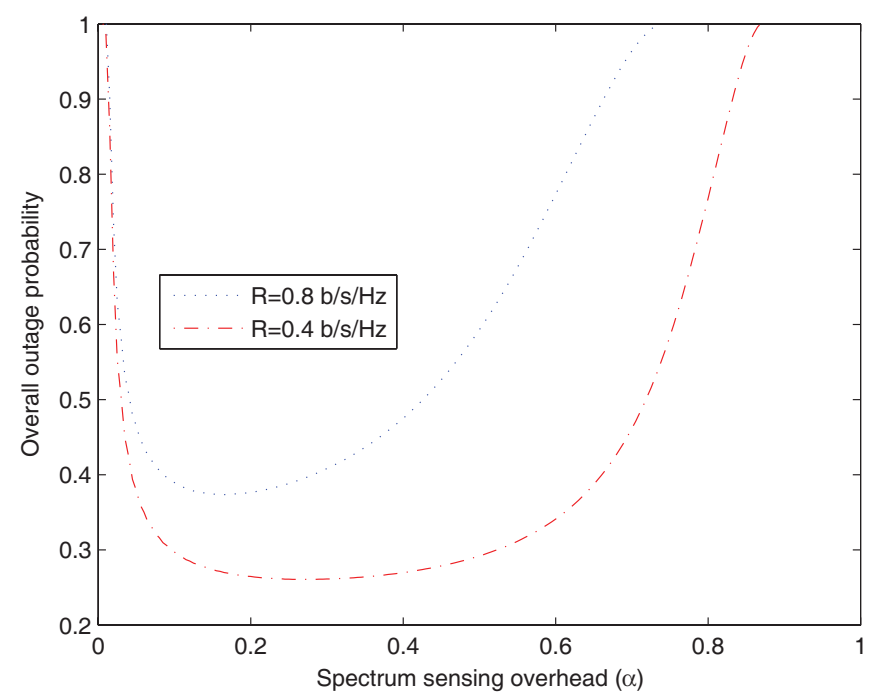

Fig. 9. Overall outage probability versus the spectrum sensing overhead $\alpha$ for different values of the data rate $R$ with $\mathrm{P}_{\mathrm{a}}=0.8, \lambda=0.1, \mathrm{P}_{\mathrm{d}_{2} \mathrm{~s}}=$ $\mathrm{P}_{\mathrm{d}, \mathrm{r}}=0.99, \gamma_{s}=10 \mathrm{~dB}, \gamma_{p}=5 \mathrm{~dB}, T=20 \mathrm{~ms}, f_{s}=25 \mathrm{kHz}, \sigma_{p s}^{2}=$ $\sigma_{p r}^{2}=\sigma_{p d}^{2}=0.5, \sigma_{s d}^{2}=1.5$, and $\sigma_{s r}^{2}=\sigma_{r d}^{2}=1$.

between the overall outage probability and spectrum sensing overhead $\alpha$ is not simply increasing or decreasing. In Fig. 9, we further show the overall outage probability versus the spectrum sensing overhead $\alpha$. As can be observed from Fig. 9, the optimal values of $\alpha$ corresponding to $R=0.4 \mathrm{~b} / \mathrm{s} / \mathrm{Hz}$ and $R=0.8 \mathrm{~b} / \mathrm{s} / \mathrm{Hz}$ are approximately equal to 0.3 and 0.15 , respectively. It is pointed out that for any given parameter set, an optimal spectrum sensing overhead $\alpha^{*}$ can be determined through numerical computation.

\section{Conclusion}

Cognitive radio has been proposed as an effective means to promote the utilization of valuable wireless spectrum resources. In this paper, we have presented a comprehensive analysis for the cognitive transmission, where a cognitive source first detects the available spectrum holes through spectrum sensing and then transmits its data over the detected holes. A closed-form expression of the overall outage probability for cognitive transmissions has been obtained to show the impact of spectrum sensing overhead. Numerical results have shown that minimum overall outage probability can be achieved through a tradeoff between the hole detection and the data transmission phases.

Furthermore, we have proposed a cognitive relay transmission scheme, in which a cognitive relay is used to assist a cognitive source for the data transmission in an adaptive manner. In particular, cognitive relay forwards its decoded result only when it can decode the received signal successfully and can detect a spectrum hole; otherwise, the cognitive source, instead of the cognitive relay, would determine whether or not to repeat the signal transmission depending on its hole detection result. Also, a closed-form expression of the overall outage probability has been derived for the proposed cognitive relay scheme. Numerical evaluations have been conducted to show the advantage of the cognitive relay transmission scheme.

\section{APPENDIX A \\ DERIVATION OF (9)}

Considering the central limit theorem (CLT), for a large number $N$, random variable $T(y)$ given $H_{p}(k, 1)=H_{0}$ follows a Gaussian distribution with mean $N_{0}$ and variance $N_{0}^{2} / N$ (see the proposition 1 in [25] for proof). Therefore, the false alarm probability $\mathrm{P}_{\mathrm{f}}(k)$ at time slot $k$ is obtained from (7) as

$$
\begin{aligned}
\mathrm{P}_{\mathrm{f}}(k) & \cong \int_{\delta}^{+\infty} \frac{\sqrt{N}}{\sqrt{2 \pi} N_{0}} \exp \left[-\frac{N\left(x-N_{0}\right)^{2}}{2 N_{0}^{2}}\right] d x \\
& =Q\left(\left(\frac{\delta}{N_{0}}-1\right) \sqrt{N}\right)
\end{aligned}
$$

where $Q(\cdot)$ is defined as

$$
Q(x)=\frac{1}{\sqrt{2 \pi}} \int_{x}^{+\infty} \exp \left(-\frac{y^{2}}{2}\right) d y
$$

Without loss of generality, we consider that the primary signal follows a complex symmetric Gaussian distribution, which a typical OFDM (orthogonal frequency-division multiplex) modulated signal [32]. Note that we can similarly extend to the other modulation types, such as the BPSK, QPSK or 16QAM modulated primary signal. According to the proposition 2 in [25], for a large number $N$, random variable $T(y)$ given $H_{p}(k, 1)=H_{1}$ follows a Gaussian distribution with mean $\left(\left|h_{p s}(k)\right|^{2} \gamma_{p}+1\right) N_{0}$ and variance $\left(\left|h_{p s}(k)\right|^{2} \gamma_{p}+1\right)^{2} N_{0}^{2} / N$, where $h_{p s}(k)$ is a fading coefficient of the channel from PU to $\mathrm{CS}$ at time slot $k$. Hence, the detection probability $\mathrm{P}_{\mathrm{d}}(k)$ at time slot $k$ is calculated from (8) as

$$
\mathrm{P}_{\mathrm{d}}(k)=Q\left(\frac{\delta \sqrt{N}}{N_{0}\left(\left|h_{p s}(k)\right|^{2} \gamma_{p}+1\right)}-\sqrt{N}\right)
$$

Given a target detection probability $\mathrm{P}_{\mathrm{d}}$ and fading coefficient $h_{p s}(k)$, the instantaneous false alarm probability $\mathrm{P}_{\mathrm{f}}(k)$ is calculated from (A.1) and (A.3) as

$$
\mathrm{P}_{\mathrm{f}}(k)=Q\left(\kappa\left|h_{p s}(k)\right|^{2}+Q^{-1}\left(\mathrm{P}_{\mathrm{d}}\right)\right)
$$


where $\kappa=\gamma_{p} Q^{-1}\left(\mathrm{P}_{\mathrm{d}}\right)+\sqrt{N} \gamma_{p}$ and $Q^{-1}(\cdot)$ is an inverse $Q(\cdot)$ function. Notice that $\mathrm{RV} X=\left|h_{p s}(k)\right|^{2}$ follows an exponential distribution with parameter $1 / \sigma_{p s}^{2}$. Therefore, the average false alarm probability $\mathrm{P}_{\mathrm{f}}$ can be calculated as

$$
\begin{aligned}
\mathrm{P}_{\mathrm{f}} & =\int_{0}^{\infty} Q\left(\kappa x+Q^{-1}\left(\mathrm{P}_{\mathrm{d}}\right)\right) \frac{1}{\sigma_{p s}^{2}} \exp \left(-\frac{x}{\sigma_{p s}^{2}}\right) d x \\
& =\iint_{\Xi} \frac{1}{\sigma_{p s}^{2}} \exp \left(-\frac{x}{\sigma_{p s}^{2}}\right) \frac{1}{\sqrt{2 \pi}} \exp \left(-\frac{y^{2}}{2}\right) d x d y
\end{aligned}
$$

where $\Xi=\left\{(x, y) \mid 0<x<\infty, \kappa x+Q^{-1}\left(\mathrm{P}_{\mathrm{d}}\right)<y<\infty\right\}$. Integrating (A.5) first with respect to $x$, then with respect to $y$, we obtain

$\mathrm{P}_{\mathrm{f}}= \begin{cases}\mathrm{P}_{\mathrm{d}}+\left[1-Q\left(Q^{-1}\left(\mathrm{P}_{\mathrm{d}}\right)+\frac{1}{\sigma_{p s}^{2} \kappa}\right)\right] \exp (\xi), & Q(-\sqrt{N})<\mathrm{P}_{\mathrm{d}} \leq 1 \\ \mathrm{P}_{\mathrm{d}}, & \mathrm{P}_{\mathrm{d}}=Q(-\sqrt{N}) \\ \mathrm{P}_{\mathrm{d}}-Q\left(Q^{-1}\left(\mathrm{P}_{\mathrm{d}}\right)+\frac{1}{\sigma_{p s}^{2} \kappa}\right) \exp (\xi), & 0 \leq \mathrm{P}_{\mathrm{d}}<Q(-\sqrt{N})\end{cases}$

where $\xi=\frac{Q^{-1}\left(\mathrm{P}_{\mathrm{d}}\right)}{\sigma_{\mathrm{ps}}^{2} \kappa}+\frac{1}{2 \sigma_{\mathrm{ps}}^{4} \kappa^{2}}$. From detection theory, for any reasonable detector, $\mathrm{P}_{\mathrm{f}}$ is always smaller than or equal to $\mathrm{P}_{\mathrm{d}}$, or else it is worse than tossing a coin. Therefore, the number of samples $N$ should satisfy

$$
N \geq\left[Q^{-1}\left(\mathrm{P}_{\mathrm{d}}\right)\right]^{2}
$$

which is due to the fact that from central limit theorem, the number of samples should be sufficiently large so that the output statistic $T(y)$ of the energy detector can be approximated to a Gaussian distribution. Combining (A.6) and (A.7) yields $\mathrm{P}_{\mathrm{f}}= \begin{cases}\mathrm{P}_{\mathrm{d}}, & \mathrm{P}_{\mathrm{d}}=Q(-\sqrt{N}) \\ \mathrm{P}_{\mathrm{d}}-Q\left(Q^{-1}\left(\mathrm{P}_{\mathrm{d}}\right)+\frac{1}{\sigma_{p s}^{2} \kappa}\right) \exp (\xi), & \text { otherwise }\end{cases}$ Note that RVs $\left|h_{s d}(k)\right|^{2}$ and $\left|h_{p d}(k)\right|^{2}$ follow the exponential distribution with parameters $1 / \sigma_{s d}^{2}$ and $1 / \sigma_{p d}^{2}$, respectively, and are independent of each other. Thus, the probability integrals given in (5) can be calculated as

$$
\operatorname{Pr}\left\{\left|h_{s d}(k)\right|^{2}<\Lambda\right\}=1-\exp \left(-\frac{\Lambda}{\sigma_{s d}^{2}}\right)
$$

and

$$
\begin{aligned}
& \operatorname{Pr}\left\{\left|h_{s d}(k)\right|^{2}-\left|h_{p d}(k)\right|^{2} \gamma_{p} \Lambda<\Lambda\right\} \\
& =\iint_{\Omega} \frac{1}{\sigma_{s d}^{2}} \exp \left(-\frac{x}{\sigma_{s d}^{2}}\right) \frac{1}{\sigma_{p d}^{2}} \exp \left(-\frac{y}{\sigma_{p d}^{2}}\right) d x d y
\end{aligned}
$$

where $\Omega=\left\{(x, y) \mid x-y \gamma_{p} \Lambda<\Lambda\right\}$. Eq. (A.10) can be derived as

$$
\begin{aligned}
& \operatorname{Pr}\left\{\left|h_{s d}(k)\right|^{2}-\left|h_{p d}(k)\right|^{2} \gamma_{p} \Lambda<\Lambda\right\} \\
& =1-\frac{\sigma_{s d}^{2}}{\sigma_{p d}^{2} \gamma_{p} \Lambda+\sigma_{s d}^{2}} \exp \left(-\frac{\Lambda}{\sigma_{s d}^{2}}\right)
\end{aligned}
$$

Substituting (A.9) and (A.11) into (5) yields (9).

\section{APPENDIX B}

\section{Calculation of (17)}

The term $\operatorname{Pr}\left\{\operatorname{SINR}_{\mathrm{d}}(\Theta=1)<\gamma_{s} \Delta, \Theta=1\right\}$ given in (29) can be rewritten as (B.1) at the top of the following page. By using conditional probabilities, (B.1) can be calculated as
(B.2), where $\mathrm{P}_{\mathrm{f}, \mathrm{s}}$ and $\mathrm{P}_{\mathrm{d}, \mathrm{s}}$ are, respectively, the probability of false alarm and the probability of detection of the primaryuser-presence by $C S$ and, moreover, $\mathrm{P}_{\mathrm{f}, \mathrm{r}}$ and $\mathrm{P}_{\mathrm{d}, \mathrm{r}}$ are the corresponding probabilities of primary user detection by CR. Similar to (A.9), it is easy to show

$\mathrm{P}_{\mathrm{f}, \mathrm{s}}=\left\{\begin{array}{l}\mathrm{P}_{\mathrm{d}, \mathrm{s}}, \quad \mathrm{P}_{\mathrm{d}, \mathrm{s}}=Q(-\sqrt{N}) \\ \mathrm{P}_{\mathrm{d}, \mathrm{s}}-Q\left(Q^{-1}\left(\mathrm{P}_{\mathrm{d}, \mathrm{s}}\right)+\frac{1}{\sigma_{p s}^{2} \kappa_{s}}\right) \exp \left(\xi_{s}\right), \text { otherwise }\end{array}\right.$

and

$\mathrm{P}_{\mathrm{f}, \mathrm{r}}=\left\{\begin{array}{l}\mathrm{P}_{\mathrm{d}, \mathrm{r}}, \quad \mathrm{P}_{\mathrm{d}, \mathrm{r}}=Q(-\sqrt{N}) \\ \mathrm{P}_{\mathrm{d}, \mathrm{r}}-Q\left(Q^{-1}\left(\mathrm{P}_{\mathrm{d}, \mathrm{r}}\right)+\frac{1}{\sigma_{p s}^{2} \kappa_{r}}\right) \exp \left(\xi_{r}\right), \text { otherwise }\end{array}\right.$

where $\kappa_{s}=\gamma_{p} Q^{-1}\left(\mathrm{P}_{\mathrm{d}, \mathrm{s}}\right)+\sqrt{N} \gamma_{p}, \quad \xi_{s}=\frac{Q^{-1}\left(\mathrm{P}_{\mathrm{d}, \mathrm{s}}\right)}{\sigma_{p s}^{2} \kappa_{s}}+$ $\frac{1}{2 \sigma_{p_{1}}^{4} \kappa_{s}^{2}}, \quad \kappa_{r}=\gamma_{p} Q^{-1}\left(\mathrm{P}_{\mathrm{d}, \mathrm{r}}\right)+\sqrt{N} \gamma_{p} \quad \xi_{r}=\frac{Q^{-1}\left(\mathrm{P}_{\mathrm{d}, \mathrm{r}}\right)}{\sigma_{p r}^{2} \kappa_{r}}+$ $\frac{p_{1}^{4}}{2 \sigma_{p r}^{4} \kappa_{r}^{2}}$, and the number of samples satisfies $N \geq$ $\max \left\{\left[\mathrm{Q}^{-1}\left(\mathrm{P}_{\mathrm{d}, \mathrm{s}}\right)\right]^{2},\left[\mathrm{Q}^{-1}\left(\mathrm{P}_{\mathrm{d}, \mathrm{r}}\right)\right]^{2}\right\}$. Clearly, the first term, $\mathrm{B} 2(\mathrm{a})$, as given in (B.2) is given by

$$
\mathrm{B} 2(\mathrm{a})=\mathrm{P}_{\mathrm{a}}\left(1-\mathrm{P}_{\mathrm{t}}\right)\left(1-\mathrm{P}_{\mathrm{f}, \mathrm{s}}\right)\left(1-\mathrm{P}_{\mathrm{f}, \mathrm{r}}\right) \exp \left(-\frac{\Delta}{\sigma_{s r}^{2}}\right) \varpi
$$

where parameter $\varpi$ is given by

$$
\varpi= \begin{cases}1-\left(1+\frac{2 \Delta}{\sigma_{s d}^{2}}\right) \exp \left(-\frac{2 \Delta}{\sigma_{s d}^{2}}\right), & \sigma_{s d}^{2}=\sigma_{r d}^{2} \\ 1-\frac{\sigma_{s d}^{2}}{\sigma_{s d}^{2}-\sigma_{r d}^{2}} \exp \left(-\frac{2 \Delta}{\sigma_{s d}^{2}}\right) & \\ -\frac{\sigma_{r d}^{2}}{\sigma_{r d}^{2}-\sigma_{s d}^{2}} \exp \left(-\frac{2 \Delta}{\sigma_{r d}^{2}}\right), & \text { otherwise }\end{cases}
$$

Also, the second term, B2(b), in (B.2) is calculated as

$$
\begin{aligned}
\mathrm{B} 2(\mathrm{~b})= & \frac{\mathrm{P}_{\mathrm{t}}\left(1-\mathrm{P}_{\mathrm{d}, \mathrm{r}}\right)}{\left(1-\mathrm{P}_{\mathrm{t}}\right)\left(1-\mathrm{P}_{\mathrm{f}, \mathrm{r}}\right)} \mathrm{B} 2(\mathrm{a}) \\
& +\mathrm{P}_{\mathrm{a}} \mathrm{P}_{\mathrm{t}}\left(1-\mathrm{P}_{\mathrm{f}, \mathrm{s}}\right)\left(1-\mathrm{P}_{\mathrm{d}, \mathrm{r}}\right) \exp \left(-\frac{\Delta}{\sigma_{s r}^{2}}\right) \vartheta
\end{aligned}
$$

where $\mathrm{B} 2(\mathrm{a})$ is given in (B.5) and the parameter $\vartheta$ is given by

$$
\vartheta= \begin{cases}\frac{\mathrm{K}}{\mathrm{H}_{s d}}\left(\frac{\mathrm{K}}{\mathrm{H}_{s d}}+\frac{2 \Delta}{\sigma_{s d}^{2}}\right) \exp \left(-\frac{2 \Delta}{\sigma_{s d}^{2}}\right), & \sigma_{s d}^{2}=\sigma_{r d}^{2} \\ \frac{\sigma_{s d}^{2} \mathrm{~K}}{\left(\sigma_{s d}^{2}-\sigma_{r d}^{2}\right) \mathrm{H}_{s d}} \exp \left(-\frac{2 \Delta}{\sigma_{s d}^{2}}\right) & \\ +\frac{\sigma_{r d}^{2} \mathrm{~K}}{\left(\sigma_{r d}^{2}-\sigma_{s d}^{2}\right) \mathrm{H}_{r d}} \exp \left(-\frac{2 \Delta}{\sigma_{r d}^{2}}\right), & \text { otherwise }\end{cases}
$$

where $\Delta=\left[2^{2 R /(1-\alpha)}-1\right] / \gamma_{s}, \mathrm{~K}=\sigma_{p d}^{2} \gamma_{p} \Delta, \mathrm{H}_{s d}=\mathrm{K}+\sigma_{s d}^{2}$ and $\mathrm{H}_{r d}=\mathrm{K}+\sigma_{r d}^{2}$. Similarly, the third term, B2(c), in (B.2) is calculated as

$$
\mathrm{B} 2(\mathrm{c})=\frac{\left(1-\mathrm{P}_{\mathrm{a}}\right)\left(1-\mathrm{P}_{\mathrm{d}, \mathrm{s}}\right)\left(1-\mathrm{P}_{\mathrm{f}, \mathrm{r}}\right) \sigma_{s r}^{2}}{\mathrm{P}_{\mathrm{a}}\left(1-\mathrm{P}_{\mathrm{f}, \mathrm{s}}\right)\left(1-\mathrm{P}_{\mathrm{d}, \mathrm{r}}\right)\left(\sigma_{s r}^{2}+\sigma_{p r}^{2} \gamma_{p} \Delta\right)} \times \mathrm{B} 2(\mathrm{~b})
$$

where B2(b) is given in (B.7). Besides, the fourth term, B2(d), as given in (B.2) is calculated as

$$
\begin{aligned}
& \mathrm{B} 2(\mathrm{~d})=\left(1-\mathrm{P}_{\mathrm{a}}\right)\left(1-\mathrm{P}_{\mathrm{t}}\right)\left(1-\mathrm{P}_{\mathrm{d}, \mathrm{s}}\right)\left(1-\mathrm{P}_{\mathrm{d}, \mathrm{r}}\right) \\
& \times \operatorname{Pr}\left\{\left|h_{s r}(k)\right|^{2}-\left|h_{p r}(k)\right|^{2} \gamma_{p} \Delta>\Delta\right\} \quad \cdots \cdots \cdots \cdots \cdots \cdot \ldots \cdot \mathrm{B} 9(\mathrm{I}) \\
& \times \operatorname{Pr}\left\{\left|h_{s d}(k)\right|^{2}+\left|h_{r d}(k)\right|^{2}-2\left|h_{p d}(k)\right|^{2} \gamma_{p} \Delta<2 \Delta\right\} \cdots \mathrm{B} 9(\mathrm{II})
\end{aligned}
$$




$$
\begin{aligned}
& \operatorname{Pr}\left\{\operatorname{SINR}_{\mathrm{d}}(\Theta=1)<\gamma_{s} \Delta, \Theta=1\right\} \\
& =\operatorname{Pr}\left\{\operatorname{SINR}_{\mathrm{d}}(\Theta=1)<\gamma_{s} \Delta, \Theta=1, H_{p}(2 k-1)=H_{0}, H_{p}(2 k)=H_{0}\right\} \\
& \quad+\operatorname{Pr}\left\{\operatorname{SINR}_{\mathrm{d}}(\Theta=1)<\gamma_{s} \Delta, \Theta=1, H_{p}(2 k-1)=H_{0}, H_{p}(2 k)=H_{1}\right\} \\
& \quad+\operatorname{Pr}\left\{\operatorname{SINR}_{\mathrm{d}}(\Theta=1)<\gamma_{s} \Delta, \Theta=1, H_{p}(2 k-1)=H_{1}, H_{p}(2 k)=H_{0}\right\} \\
& \quad+\operatorname{Pr}\left\{\operatorname{SINR}_{\mathrm{d}}(\Theta=1)<\gamma_{s} \Delta, \Theta=1, H_{p}(2 k-1)=H_{1}, H_{p}(2 k)=H_{1}\right\}
\end{aligned}
$$

$$
\begin{aligned}
\operatorname{Pr} & \left\{\operatorname{SINR}_{\mathrm{d}}(\Theta=1)<\gamma_{s} \Delta, \Theta=1\right\} \\
= & \mathrm{P}_{\mathrm{a}}\left(1-\mathrm{P}_{\mathrm{t}}\right)\left(1-\mathrm{P}_{\mathrm{f}, \mathrm{s}}\right)\left(1-\mathrm{P}_{\mathrm{f}, \mathrm{r}}\right) \operatorname{Pr}\left\{\left|h_{s d}(k)\right|^{2}+\left|h_{r d}(k)\right|^{2}<2 \Delta,\left|h_{s r}(k)\right|^{2}>\Delta\right\} \\
& +\mathrm{Pa}_{\mathrm{a}}\left(1-\mathrm{P}_{\mathrm{f}, \mathrm{s}}\right)\left(1-\mathrm{P}_{\mathrm{d}, \mathrm{r}}\right) \operatorname{Pr}\left\{\left|h_{s d}(k)\right|^{2}+\left|h_{r d}(k)\right|^{2}-\left|h_{p d}(k)\right|^{2} \gamma_{p} \Delta<2 \Delta,\left|h_{s r}(k)\right|^{2}>\Delta\right\} \\
& +\left(1-\mathrm{P}_{\mathrm{a}}\right) \mathrm{P}_{\mathrm{t}}\left(1-\mathrm{P}_{\mathrm{d}, \mathrm{s}}\right)\left(1-\mathrm{P}_{\mathrm{f}, \mathrm{r}}\right) \operatorname{Pr}\left\{\begin{array}{l}
\left|h_{s d}(k)\right|^{2}+\left|h_{r d}(k)\right|^{2}-\left|h_{p d}(k)\right|^{2} \gamma_{p} \Delta<2 \Delta, \\
\left|h_{s r}(k)\right|^{2}-\left|h_{p r}(k)\right|^{2} \gamma_{p} \Delta>\Delta
\end{array}\right\} \\
& +\left(1-\mathrm{P}_{\mathrm{a}}\right)\left(1-\mathrm{P}_{\mathrm{t}}\right)\left(1-\mathrm{P}_{\mathrm{d}, \mathrm{s}}\right)\left(1-\mathrm{P}_{\mathrm{d}, \mathrm{r}}\right) \operatorname{Pr}\left\{\begin{array}{l}
\left|h_{s d}(k)\right|^{2}+\left|h_{r d}(k)\right|^{2}-2\left|h_{p d}(k)\right|^{2} \gamma_{p} \Delta<2 \Delta, \\
\left|h_{s r}(k)\right|^{2}-\left|h_{p r}(k)\right|^{2} \gamma_{p} \Delta>\Delta
\end{array}\right\}
\end{aligned}
$$

where B9(I) is calculated as

$$
\mathrm{B} 9(\mathrm{I})=\frac{\sigma_{s r}^{2}}{\sigma_{p r}^{2} \gamma_{p} \Delta+\sigma_{s r}^{2}} \exp \left(-\frac{\Delta}{\sigma_{s r}^{2}}\right)
$$

Furthermore, B9(II) can be derived as

$$
\mathrm{B} 9(\mathrm{II})=\varpi+\tilde{\vartheta}
$$

where the parameter $\varpi$ is given by (B.6) and $\tilde{\vartheta}$ is

$$
\tilde{\vartheta}= \begin{cases}\frac{\tilde{\mathrm{K}}}{\tilde{\mathrm{H}}_{s d}}\left(\frac{\tilde{\mathrm{K}}}{\tilde{\mathrm{H}}_{s d}}+\frac{2 \Delta}{\sigma_{s d}^{2}}\right) \exp \left(-\frac{2 \Delta}{\sigma_{s d}^{2}}\right), & \sigma_{s d}^{2}=\sigma_{r d}^{2} \\ \frac{\sigma_{s d}^{2} \tilde{\mathrm{K}}}{\left(\sigma_{s d}^{2}-\sigma_{r d}^{2}\right) \tilde{\mathrm{H}}_{s d}} \exp \left(-\frac{2 \Delta}{\sigma_{s d}^{2}}\right) & \\ +\frac{\sigma_{r d}^{2} \tilde{\mathrm{K}}}{\left(\sigma_{r d}^{2}-\sigma_{s d}^{2}\right) \tilde{\mathrm{H}}_{r d}} \exp \left(-\frac{2 \Delta}{\sigma_{r d}^{2}}\right), & \text { otherwise }\end{cases}
$$

where $\tilde{\mathrm{K}}=2 \sigma_{p d}^{2} \gamma_{p} \Delta, \tilde{\mathrm{H}}_{s d}=\tilde{\mathrm{K}}+\sigma_{s d}^{2}$ and $\tilde{\mathrm{H}}_{r d}=\tilde{\mathrm{K}}+\sigma_{r d}^{2}$. According to the definition of the case $\Theta=2$ as given in (25), we have

$$
\begin{aligned}
& \operatorname{Pr}\left\{\operatorname{SINR}_{\mathrm{d}}(\Theta=2)<\gamma_{s} \Delta, \Theta=2\right\} \\
& =\operatorname{Pr}\left\{\operatorname{SINR}_{\mathrm{d}}(\Theta=2)<\gamma_{s} \Delta\right\} \cdots \cdots \cdots \mathrm{B} 12(\mathrm{a}) \\
& -\operatorname{Pr}\left\{\operatorname{SINR}_{\mathrm{d}}(\Theta=2)<\gamma_{s} \Delta, \Theta=1\right\} \cdots \mathrm{B} 12(\mathrm{~b})
\end{aligned}
$$

Using conditional probabilities, the terms B12(a) and B12(b) can be calculated as (B.13) and (B.14), respectively, at the top of the following page. and Note that we can easily obtain the closed-form expressions of all the probability integrals as given in (B.13) and (B.14). We have now derived the closed-form expressions for the terms $\operatorname{Pr}\left\{\operatorname{SINR}_{\mathrm{d}}(\Theta=1)<\gamma_{s} \Delta, \Theta=1\right\}$ and $\operatorname{Pr}\left\{\operatorname{SINR}_{d}(\Theta=2)<\gamma_{s} \Delta, \Theta=2\right\}$ as given in (B.2) and (B.12), respectively.

\section{ACKNOWLEDGMENT}

The authors are grateful to the anonymous reviewers for their valuable comments and constructive suggestions that have indeed helped improve this paper significantly.

\section{REFERENCES}

[1] Federal Communications Commission (FCC), ET Docket No 03-322 Notice of Proposed Rule Making and Order, Dec. 2003.

[2] J. Mitola and G. Q. Maguire, "Cognitive radio: making software radios more personal," IEEE Personal Commun., vol. 6, pp. 13-18, 1999.

[3] S. Haykin, "Cognitive radio: brain-empowered wireless communications," IEEE J. Sel. Areas Commun., vol. 23, no. 2, pp. 201-220, 2005.

[4] F. Akyildiz, W. Y. Lee, M. C. Vuran, and S. Mohanty, "NeXt generation/dynamic spectrum access/cognitive radio wireless networks: a survey," Computer Networks, vol. 50, pp. 2127-2159, 2006.

[5] H. Urkowitz, "Energy detection of unknown deterministic signals," Proc. IEEE, vol. 55, no. 4, pp. 523-531, 1967.

[6] V. I. Kostylev, "Energy detection of a signal with random amptitude," in Proc. IEEE International Conference on Communications, vol. 3, pp. 1606-1610, 2002.

[7] A. Sahai, N. Hoven, and R. Tandra, "Some fundamental limits in cognitive radio," in Proc. Allerton Conf. on Commun., Control and Computing, 2004.

[8] S. M. Kay, Fundamentals of Statistical Signal Processing: Detection Theory. Upper Saddle River, NJ: Prentice Hall, 1998.

[9] A. Fehske, J. D. Gaeddert, and J. H. Reed, "A new approach to signal classification using spectral correlation and neural networks," in Proc. IEEE DySPAN, pp. 144-150, Nov. 2005.

[10] P. D. Sutton, K. E. Nolan, and L. E. Doyle, "Cyclostationary signatures in practical cognitive radio applications," IEEE J. Sel. Areas Commun., vol. 26, no. 1, pp. 13-24, 2008.

[11] A. Ghasemi and E. S. Sousa, "Collaborative spectrum sensing for opportunistic access in fading environment," in Proc. IEEE DYSPAN, pp. 131-136, 2005.

[12] G. Ganesan and Y. G. Li, "Cooperative spectrum sensing in cognitive radio-part I: two user networks," IEEE Trans. Wireless Commun., vol. 6, no. 6, pp. 2204-2213, 2007.

[13] J. Zhu, Y. Zou, and B. Zheng, "Cooperative detection for primary user in cognitive radio networks," EURASIP J. Wireless Commun. and Networking, 2009, doi:10.1155/2009/617320.

[14] R. W. Pabst and B. H. Shultz, et al., "Relay-based deployment concepts for wireless and mobile broadband radio," IEEE Commun. Mag., vol. 42, no. 9, pp. 80-89, Sep. 2004.

[15] A. J. Paulraj, D. A. Gore, R. U. Nabar, and H. Bolcskei, "An overview of MIMO communications-a key to gigabit wireless," Proc. IEEE, vol. 92, no. 2, pp. 198-218, Feb. 2004.

[16] J. N. Laneman and G. W. Wornell, "Distributed space-time-coded protocols for exploiting cooperative diversity in wireless networks," IEEE Trans. Inf. Theory, vol. 49, no. 10, pp. 2415-2425, Oct. 2003.

[17] A. Sendonaris, E. Erkip, and B. Aazhang, "User cooperation diversitypart I: system description," IEEE Trans. Commun., vol. 51, no. 11, pp. 1927-1938, 2003.

[18] A. Nosratinia and T. E. Hunter, "Cooperative communication in wireless networks," IEEE Commun. Mag., vol. 42, no.10, pp. 74-80, 2004. 


$$
\begin{aligned}
\mathrm{B} 12(\mathrm{a})= & \mathrm{P}_{\mathrm{a}}\left(1-\mathrm{P}_{\mathrm{t}}\right)\left(1-\mathrm{P}_{\mathrm{f}, \mathrm{s}}\right)^{2} \operatorname{Pr}\left\{\left|h_{s d}(k)\right|^{2}<\Delta\right\} \\
& +\left(1-\mathrm{P}_{\mathrm{a}}\right)\left(1-\mathrm{P}_{\mathrm{t}}\right)\left(1-\mathrm{P}_{\mathrm{d}, \mathrm{s}}\right)^{2} \operatorname{Pr}\left\{\left|h_{s d}(k)\right|^{2}-\left|h_{p d}(k)\right|^{2} \gamma_{p} \Delta<\Delta\right\} \\
& +\mathrm{P}_{\mathrm{t}}\left(1-\mathrm{P}_{\mathrm{f}, \mathrm{s}}\right)\left(1-\mathrm{P}_{\mathrm{d}, \mathrm{s}}\right) \operatorname{Pr}\left\{2\left|h_{s d}(k)\right|^{2}-\left|h_{p d}(k)\right|^{2} \gamma_{p} \Delta<2 \Delta\right\} \\
& +2 \mathrm{P}_{\mathrm{a}}\left(1-\mathrm{P}_{\mathrm{t}}\right)\left(1-\mathrm{P}_{\mathrm{f}, \mathrm{s}}\right) \mathrm{P}_{\mathrm{f}, \mathrm{s}} \operatorname{Pr}\left\{\left|h_{s d}(k)\right|^{2}<2 \Delta\right\} \\
& +2\left(1-\mathrm{P}_{\mathrm{a}}\right)\left(1-\mathrm{P}_{\mathrm{t}}\right)\left(1-\mathrm{P}_{\mathrm{d}, \mathrm{s}}\right) \mathrm{P}_{\mathrm{d}, \mathrm{s}} \operatorname{Pr}\left\{\left|h_{s d}(k)\right|^{2}-2\left|h_{p d}(k)\right|^{2} \gamma_{p} \Delta<2 \Delta\right\} \\
& +\mathrm{P}_{\mathrm{t}}\left(\mathrm{P}_{\mathrm{d}, \mathrm{s}}+\mathrm{P}_{\mathrm{f}, \mathrm{s}}-2 \mathrm{P}_{\mathrm{f}, \mathrm{s}} \mathrm{P}_{\mathrm{d}, \mathrm{s}}\right) \operatorname{Pr}\left\{\left|h_{s d}(k)\right|^{2}-\left|h_{p d}(k)\right|^{2} \gamma_{p} \Delta<2 \Delta\right\} \\
& +\mathrm{P}_{\mathrm{a}}\left(1-\mathrm{P}_{\mathrm{t}}\right) \mathrm{P}_{\mathrm{f}, \mathrm{s}}^{2}+\mathrm{P}_{\mathrm{t}} \mathrm{P}_{\mathrm{d}, \mathrm{s}} \mathrm{P}_{\mathrm{f}, \mathrm{s}}+\left(1-\mathrm{P}_{\mathrm{a}}\right)\left(1-\mathrm{P}_{\mathrm{t}}\right) \mathrm{P}_{\mathrm{d}, \mathrm{s}}^{2}
\end{aligned}
$$

$$
\begin{aligned}
\mathrm{B} 12(\mathrm{~b})= & \mathrm{P}_{\mathrm{a}}\left(1-\mathrm{P}_{\mathrm{t}}\right)\left(1-\mathrm{P}_{\mathrm{f}, \mathrm{s}}\right)^{2}\left(1-\mathrm{P}_{\mathrm{f}, \mathrm{r}}\right) \operatorname{Pr}\left\{\left|h_{s d}(k)\right|^{2}<\Delta\right\} \operatorname{Pr}\left\{\left|h_{s r}(k)\right|^{2}>\Delta\right\} \\
+ & \mathrm{P}_{\mathrm{a}} \mathrm{P}_{\mathrm{t}}\left(1-\mathrm{P}_{\mathrm{f}, \mathrm{s}}\right)\left(1-\mathrm{P}_{\mathrm{d}, \mathrm{s}}\right)\left(1-\mathrm{P}_{\mathrm{d}, \mathrm{r}}\right) \operatorname{Pr}\left\{2\left|h_{s d}(k)\right|^{2}-\left|h_{p d}(k)\right|^{2} \gamma_{p} \Delta<2 \Delta\right\} \\
& \times \operatorname{Pr}\left\{\left|h_{s r}(k)\right|^{2}>\Delta\right\} \\
+ & \left(1-\mathrm{P}_{\mathrm{a}}\right) \mathrm{P}_{\mathrm{t}}\left(1-\mathrm{P}_{\mathrm{f}, \mathrm{s}}\right)\left(1-\mathrm{P}_{\mathrm{d}, \mathrm{s}}\right)\left(1-\mathrm{P}_{\mathrm{f}, \mathrm{r}}\right) \operatorname{Pr}\left\{2\left|h_{s d}(k)\right|^{2}-\left|h_{p d}(k)\right|^{2} \gamma_{p} \Delta<2 \Delta\right\} \\
& \times \operatorname{Pr}\left\{\left|h_{s r}(k)\right|^{2}-\left|h_{p r}(k)\right|^{2} \gamma_{p} \Delta>\Delta\right\} \\
+ & \left(1-\mathrm{P}_{\mathrm{a}}\right)\left(1-\mathrm{P}_{\mathrm{t}}\right)\left(1-\mathrm{P}_{\mathrm{d}, \mathrm{s}}\right)^{2}\left(1-\mathrm{P}_{\mathrm{d}, \mathrm{r}}\right) \operatorname{Pr}\left\{\left|h_{s d}(k)\right|^{2}-\left|h_{p d}(k)\right|^{2} \gamma_{p} \Delta<\Delta\right\} \\
& \times \operatorname{Pr}\left\{\left|h_{s r}(k)\right|^{2}-\left|h_{p r}(k)\right|^{2} \gamma_{p} \Delta>\Delta\right\} \\
+ & \mathrm{P}_{\mathrm{a}}\left(1-\mathrm{P}_{\mathrm{t}}\right)\left(1-\mathrm{P}_{\mathrm{f}, \mathrm{s}}\right) \mathrm{P}_{\mathrm{f}, \mathrm{s}}\left(1-\mathrm{P}_{\mathrm{f}, \mathrm{r}}\right) \operatorname{Pr}\left\{\left|h_{s d}(k)\right|^{2}<2 \Delta\right\} \operatorname{Pr}\left\{\left|h_{s r}(k)\right|^{2}>\Delta\right\} \\
+ & \mathrm{P}_{\mathrm{a}} \mathrm{P}_{\mathrm{t}}\left(1-\mathrm{P}_{\mathrm{f}, \mathrm{s}}\right) \mathrm{P}_{\mathrm{d}, \mathrm{s}}\left(1-\mathrm{P}_{\mathrm{d}, \mathrm{r}}\right) \operatorname{Pr}\left\{\left|h_{s d}(k)\right|^{2}-\left|h_{p d}(k)\right|^{2} \gamma_{p} \Delta<2 \Delta\right\} \\
& \times \operatorname{Pr}\left\{\left|h_{s r}(k)\right|^{2}>\Delta\right\} \\
+ & \left(1-\mathrm{P}_{\mathrm{a}}\right) \mathrm{P}_{\mathrm{t}}\left(1-\mathrm{P}_{\mathrm{d}, \mathrm{s}}\right) \mathrm{P}_{\mathrm{f}, \mathrm{s}}\left(1-\mathrm{P}_{\mathrm{f}, \mathrm{r}}\right) \operatorname{Pr}\left\{\left|h_{s d}(k)\right|^{2}-\left|h_{p d}(k)\right|^{2} \gamma_{p} \Delta<2 \Delta\right\} \\
& \times \operatorname{Pr}\left\{\left|h_{s r}(k)\right|^{2}-\left|h_{p r}(k)\right|^{2} \gamma_{p} \Delta>\Delta\right\} \\
+ & \left(1-\operatorname{P}{ }_{\mathrm{a}}\right)\left(1-\mathrm{P}_{\mathrm{t}}\right)\left(1-\mathrm{P}_{\mathrm{d}, \mathrm{s}}\right) \mathrm{P}_{\mathrm{d}, \mathrm{s}}\left(1-\operatorname{P} \mathrm{P}_{\mathrm{d}, \mathrm{r}}\right) \operatorname{Pr}\left\{\left|h_{s d}(k)\right|^{2}-2\left|h_{p d}(k)\right|^{2} \gamma_{p} \Delta<2 \Delta\right\} \\
& \times \operatorname{Pr}\left\{\left|h_{s r}(k)\right|^{2}-\left|h_{p r}(k)\right|^{2} \gamma_{p} \Delta>\Delta\right\}
\end{aligned}
$$

[19] T. E. Hunter, S. Sanayei, and A. Nosratinia. "Outage analysis of coded cooperation," IEEE Trans. Inf. Theory, vol. 52, no. 2, pp. 375-391, 2006.

[20] A. Bletsas, A. Khisti, et al., "A simple cooperative diversity method based on network path selection," IEEE J. Sel. Areas Commun., vol. 24, no. 3, pp. 659-672, 2006.

[21] Y. Zou, B. Zheng, and J. Zhu, "Outage analysis of opportunistic cooperation over Rayleigh fading channels," IEEE Trans. Wireless Commun., vol. 8, no. 6, pp. 3077-3385, June 2009.

[22] Y. Zou, B. Zheng, and W. P. Zhu, "An opportunistic cooperation and its BER analysis," IEEE Trans. Wireless Commun., vol. 8, no. 9, pp. 4492-4497, Sep. 2009.

[23] Y. Zou, J. Zhu, B. Zheng, and Y.-D. Yao, "An adaptive cooperation diversity scheme with best-relay selection in cognitive radio networks," accepted to appear in IEEE Trans. Signal Process., 2010.

[24] A. Ghasemi and E. S. Sousa, "Optimization of spectrum sensing for opportunistic spectrum access in cognitive radio networks," in Proc. Consumer Communications and Networking Conf. (CCNC 2007), Jan. 2007, pp. 1022-1026.

[25] Y.-C. Liang, Y. Zeng, E. Peh, and A. T. Hoang, "Sensing-throughput tradeoff for cognitive radio networks," IEEE Trans. Wireless Commun., vol. 7, no. 4, pp. 1326-1337, Apr. 2008.

[26] Y. Pei, A. T. Hoang, and Y.-C. Liang, "Sensing-throughput tradeoff in cognitive radio networks: how frequently should spectrum sensing be carried out?" in Proc. IEEE Int. Symp. Personal, Indoor and Mobile Radio Commun. (PIMRC 2007), Sep. 2007, pp. 1-5.

[27] W.-Y. Lee and I. F. Akyildiz, "Optimal spectrum sensing framework for cognitive radio networks," IEEE Trans. Wireless Commun., vol. 7, no. 10, pp. 3845-3857, Oct. 2008.

[28] C.-H. Huang, Y.-C. Lai, and K.-C. Chen, "Network capacity of cognitive radio relay network," Physical Commun., vol. 1, no. 2, June 2008.

[29] J. Jia, J. Zhang, and Q. Zhang, "Cooperative relay for cognitive radio networks," in Proc. IEEE INFOCOM 2009, pp. 2304-2312.

[30] H. V. Poor, An Introduction to Signal Detection and Estimation. Springer-Verlag, 1994.

[31] C. E. Shannon, "A mathmatical theroy of communication," Bell System Techn. J., vol. 27, pp. 379-423, 1948.

[32] S. Masoud, G.-A. Mohammad, and H. K. Babak, "On the peak-toaverage power of OFDM signals based on oversampling," IEEE Trans. Commun., vol. 51, no. 1, pp. 72-78, Jan. 2003. 


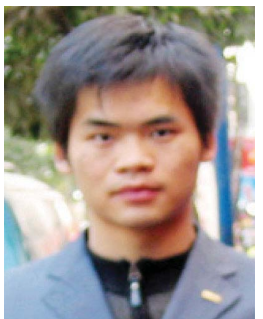

Yulong Zou received his B.Eng. degree (with the highest honor) in Information Engineering from Nanjing University of Posts and Telecommunications (NUPT), Nanjing China, in 2006. He is currently working toward his Ph.D. degrees, respectively, at the Institute of Signal Processing and Transmission of NUPT, Nanjing China, and the Electrical and Computer Engineering Department of Stevens Institute of Technology (SIT), NJ USA. His research interests span the broad area of scalable wireless communication and networking, with emphasis on cooperative relay technology. Recently, he focuses on the investigation of cooperative relay techniques in cognitive radio networks.

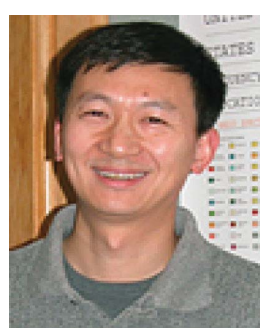

Yu-Dong Yao (S'88-M'88-SM'94) has been with Stevens Institute of Technology, Hoboken, New Jersey, since 2000 and is currently a professor and department director of electrical and computer engineering. He is also a director of Stevens' Wireless Information Systems Engineering Laboratory (WISELAB). Previously, from 1989 and 1990, he was at Carleton University, Ottawa, Canada, as a Research Associate working on mobile radio communications. From 1990 to 1994, he was with Spar Aerospace Ltd., Montreal, Canada, where he was involved in research on satellite communications. From 1994 to 2000, he was with Qualcomm Inc., San Diego, CA, where he participated in research and development in wireless code-division multiple-access (CDMA) systems.
He holds one Chinese patent and twelve U.S. patents. His research interests include wireless communications and networks, spread spectrum and CDMA, antenna arrays and beamforming, cognitive and software defined radio (CSDR), and digital signal processing for wireless systems. Dr. Yao was an Associate Editor of IEEE Communications Letters and IEEE Transactions on Vehicular Technology, and an Editor for IEEE Transactions on Wireless Communications. He received the B.Eng. and M.Eng. degrees from Nanjing University of Posts and Telecommunications, Nanjing, China, in 1982 and 1985, respectively, and the Ph.D. degree from Southeast University, Nanjing, China, in 1988, all in electrical engineering.

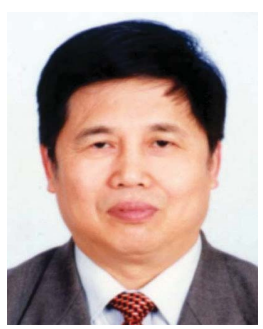

Baoyu Zheng received B.S. and M.S. degrees from the Department of Circuit and Signal System, NUPT, in 1969 and 1981, respectively. Since then, he has been engaged in teaching and researching at Signal and Information Processing. He is a full professor and doctoral advisor at NUPT. His research interests span the broad area of the intelligent signal processing, wireless network and signal processing for modern communication, and the quantum signal processing. 